\title{
CD40 provides immune privilege to the bone marrow hematopoietic niche
}

Short Title: CD40 controls bone marrow immune homeostasis

Barbara Bassani ${ }^{1}$, Alessandro Gulino ${ }^{2}$, Paola Portararo ${ }^{1}$, Laura Botti $^{1}$, Barbara Cappetti ${ }^{1}$,

Claudia Chiodoni ${ }^{1}$, Niccolò Bolli ${ }^{3,4}$, Marilena Ciciarello ${ }^{5}$, Korinna Joehrens ${ }^{6}$, Ioannis

Anagnostopoulos $^{6}, \mathrm{Il}_{-} \mathrm{Kang} \mathrm{Na}^{7,8,9}$, Antonio Curti ${ }^{5}$, Claudio Tripodo ${ }^{2}$, Mario P. Colombo ${ }^{1}$,

Sabina Sangaletti ${ }^{1 *}$.

${ }^{1}$ Department of Research, Fondazione IRCCS Istituto Nazionale Tumori, Milan, Italy

${ }^{2}$ University of Palermo, Palermo, Italy

${ }^{3}$ Department of Oncology and Hemato-Oncology, University of Milan, Milan, Italy

4 Department of Oncology and Hematology, Fondazione IRCCS Istituto Nazionale dei Tumori, Milan, Italy

${ }^{5}$ Department of Experimental, Diagnostic and Specialty Medicine - DIMES, Institute of Hematology " Seràgnoli", Bologna, Italy

${ }^{6}$ Institute of Pathology, Charité-Universitätsmedizin Berlin, 10117, Berlin, Germany

${ }^{7}$ Department of Hematology, Oncology and Tumor Immunology, Charité Universitätsmedizin Berlin, corporate member of Freie Universität Berlin, HumboldtUniversität zu Berlin, and Berlin Institute of Health, Berlin, Germany

${ }^{8}$ Experimental and Clinical Research Center, Berlin, Germany; Berlin-Brandenburg Center for Regenerative Therapies, Berlin, Germany

${ }^{9}$ Berlin Institute of Health, Berlin, Germany

BB and AG are co-first authors; SS and MPC are co-senior authors

Correspondence and requests for materials should be addressed to Sabina Sangaletti sabina.sangaletti@istitutotumori.mi.it. Address: Fondazione IRCCS Istituto Nazionale dei Tumori, 20133, Milan, Italy.

Word Count: main text 4675; Abstract 195; Figures 7; References 40 


\title{
Key points
}

1. CD40 regulates BM immunological tolerance following total body irradiation (TBI) and transplantation (BMT).

2. Loss of CD40 ${ }^{+}$MHC- ${ }^{\text {high }}{ }^{-}$BM-MSCs is associated to BM manifestation of aGVHD in human and murine model.

\begin{abstract}
Allogeneic bone marrow transplantation remains the only therapeutic option for a wide range of hematological malignancies despite the risk of possible adverse, immune-related events, such as infection and acute graft-versus-host disease (aGVHD). aGVHD is characterized by T-cell activation, defective B-cell development and osteoblastic niche destruction in bone marrow (BM) among other issues. Transplant conditioning regimens cause excessive inflammatory cytokines production and impaired regulatory T-cell control of aberrant T-cell activation. Here, we show that mesenchymal cells (MSCs) upregulated CD40 upon irradiation at the expense of mesenchymal markers, and that CD40 endows MSC of regulatory function on Treg homeostasis and fitness. Transplantation of wild type hematopoietic cells into a CD40-null recipient reduces Treg numbers allowing persistent Tcell activation and pro-inflammatory cytokines production causing, impaired Blymphopoiesis. These evidences find correlation in aGVHD patients showing the loss of CD40+ BM-MSCs along with reduction in cells of the B-lineage. Modeling aGVHD in mice we show that the elimination of CD40+ BM-MSCs relies on their higher expression of MHCI molecules. Indeed, aGVHD mice compared to MHC-matched controls showed the loss of MHC-I + radio-resistant host BM-MSCs. Our data point to CD40+ MHC-I+BM-MSCs as a key regulator of $\mathrm{BM}$ tolerogenic niches.
\end{abstract}




\section{Introduction}

Allogeneic bone marrow transplantation (BMT) remains the only therapeutic option for a wide range of hematological malignancies. However, it carries a significant risk of morbidity and mortality, which are mostly linked to adverse, immune-related events, such as infection and acute graft-versus-host disease (aGVHD). Thus, a better understanding of the mechanisms underlying immune regulation during bone marrow (BM) transplantation would significantly expand its application and potential benefits.

The BM has the features of an immune-privileged organ. This condition is granted by its peculiar enrichment in Foxp3+ regulatory T-cells (Tregs), which represent up to $30-40 \%$ of the total CD4 T-cell population, exceeding the fraction of Tregs in other lymphoid organs by 3- to 4-fold. In the BM, Tregs populate hematopoietic stem cell (HSC) niches where they protect endogenous HSCs from excessive inflammation and, during BMT, enable transplanted allogeneic HSCs to avoid rejection ${ }^{12}$. Indeed, an excess of inflammatory cytokines, such as interferon (IFN) or tumor necrosis factor (TNF), can induce HSC dysfunction and hamper HSC differentiation into mature myelo-lymphoid lineages. After BMT, B-cells are particularly sensitive to the inflammatory conditions within the microenvironment and largely depend on the Tregs for proper reconstitution. In fact, B-cell lymphopoiesis depends on interleukin (IL)-7, which is defective in Treg-depleted mice ${ }^{3}$. Furthermore, Treg depletion unleashes the production of granulocyte-macrophage colonystimulating factor (GM-CSF), IL-6, and TNF, which promote granulopoiesis at the expense of B lymphopoiesis ${ }^{4}$. Also IFN- $\gamma$ negatively affects B-cell lymphopoiesis ${ }^{4,5}$, suggesting that BM microenvironmental inflammatory state largely depend on Treg surveillance.

Tregs regulation has been largely studies in different tissues, but less so in the BM microenvironment. In the periphery, Treg development depends on transforming growth factor (TGF)- $\beta$ and IL-2, while in the thymus IL-2 is dispensable because other coexisting cytokines can act on the IL-2R $\beta$ chain ${ }^{6}$. Further, the cluster of differentiation (CD)40/CD40 ligand (L) axis also contributes to the peripheral Treg pool ${ }^{7}$.

Bone marrow mesenchymal stroma cells (BM-MSCs) nurse HSCs and progenitor cells through the stages of quiescence/self-renewal and differentiation ${ }^{8}$. In addition to supporting HSC, BM-MSCs also exert immunoregulatory functions: they can inhibit the proliferation of T, B and natural killer (NK) lymphocytes ${ }^{9}$ and promote the conversion of Teff to Treg, in vitro. On this line, in vivo administration of BM-MSCs has beneficial effects in the treatment 
of aGVHD ${ }^{10}$. Beside the production of regulatory metabolic enzymes like indoleamine-2,3dioxygenase (IDO) ${ }^{11}$, the repertoire of co-stimulatory and/or co-inhibitory molecules harnessing BM-MSCs for immune regulation, including the role of CD40, is largely unknown. The lack of CD40 expression in normal BM and its upregulation on BM-MSCs of patients affected by splenic marginal zone lymphoma ${ }^{12}$, suggest that BM-MSC can gain immunoregulatory properties in response to microenvironment perturbation.

In this study, we evaluated the role of CD40 on BM-MSCs in sustaining a BM tolerogenic state in the context of pro-inflammatory signals preventing unwanted T-cell activation that could negatively impact on hematopoiesis. 


\section{Methods}

Patients. This study included bone marrow biopsies from 12 adult acute leukemia patients undergoing allo-HSCT at the Charité university hospital.

The patients' clinical characteristics and GVHD prophylaxis are summarized in Table 1. The study was approved by the Charité-Berlin local ethics committee. Patients gave informed consent. The study was conducted in accordance with the Declaration of Helsinki"

Mice. BALB/cAnNCrl and C57BL/6 mice were purchased from Charles River Laboratories (Calco, Italy). Tnfrsf5 ( $C d 40$ )-KO mice (on a BALB/c background) were already available in our lab, while those on a C57BL/6 background were obtained from Dr. Dellabona Paolo (San Raffaele Hospital, Milan, Italy) and Prof. Bronte Vincenzo (University of Verona, Verona, Italy). CxB6F1 (Cd40)-KO mice were obtained by crossing the $C d 40-\mathrm{KO}$ on the BALB/c background with those on the C57BL/6 background. All experiments involving animals described in this study were approved by the Ministry of Health (authorization number $\mathrm{n}$. 601/2019-PR).

BMT and aGVHD mouse experiments. Canonical BMT experiments were performed by transplanting $2 \times 10^{5}$ lin- cells into lethally irradiated WT and Cd40-KO mice as previously described ${ }^{13}$. MHC-mismatched BMT was performed transplanting lethally irradiated $\mathrm{BALB} / \mathrm{c}$ mice with $2 \times 10^{6} \mathrm{~T}$-cell-depleted BM cells from C57BL/6 mice in the presence or absence of Teff cells $\left(2 \times 10^{5}\right)$ from the same donors. T-cell-depleted BM was obtained by flushing the BM cells from donors and incubating them with $\alpha$-CD5 (Ly-1) microbeads from Miltenyi. Mice were assessed daily using a GVHD scoring system that measures items related to the known clinical signs of GVHD, including weight loss, posture, activity, fur texture, skin integrity, and diarrhea.

Flow cytometry. The composition of the B-cell compartment in the BM and spleens of BM chimeras was analyzed as previously described ${ }^{14}$. Notably, B-cell development in the BM has been classified into sequential subsets designated Fractions A, B, C, C', D, E, and F as originally described by Hardy et al. ${ }^{15}$.

Surface staining was performed in phosphate-buffered saline (PBS) supplemented with $2 \%$ fetal bovine serum (FBS) for $30 \mathrm{~min}$ on ice. Foxp3 intracellular staining was performed according to the manufacturer's instructions (eBioscience). Before IFN- $\gamma$ staining, the cells 
were stimulated in vitro for $4 \mathrm{~h}$ at $37^{\circ} \mathrm{C}$ with Cell Stimulation Cocktail plus protein transport inhibitors (eBioscience).

All antibodies are listed in Supplemental Table 2. Flow cytometry data were acquired on a LSRFortessa (Becton Dickinson) and analyzed with FlowJo software (version 8.8.6, Tree Star Inc.).

Isolation and culture of murine BM-MSCs. Murine BM-MSC cultures were obtained from the trabecular fraction of femurs and tibias of WT and CD40-KO mice. Briefly, the cellular fraction of the femurs and tibias was washed out and the compact bone was incubated with collagenase $\mathrm{I}(1 \mathrm{mg} / \mathrm{ml})$ for $1 \mathrm{~h}$ at $37^{\circ} \mathrm{C}$. After enzyme digestion, the bone suspension was passed through a 70-mm filter mesh to remove any bone spicules and large tissues. Cells were seeded in complete medium at a density of $25 \times 10^{6}$ cells $/ \mathrm{ml}$. Floating cells were removed every 3-4 days. Adherent cells were phenotypically characterized using the following antibodies: CD31, CD45, CD34, Ter119, CD44, Sca-1, and CD117. In vitro and in vivo experiments involving murine BM-MSCs were performed using cells between the 2nd and 5 th passages.

Statistical analysis. In transplantation analysis an ordinary one-way ANOVA was used, while for all the other comparisons the Unpaired t-test was used. Error bars in all graphs represent mean and standard deviation. All statistical analyses were performed with Prism 6 (GraphPad Software). 


\section{Results}

Lethal irradiation perturbs BM T-cell homeostasis and promotes the expression of CD40 in stromal cells.

We previously shown that CD40 expression characterizes stromal cells of patients who had the $\mathrm{BM}$ infiltrated by splenic marginal zone lymphoma. In these patient CD40 promotes a pro-inflammatory loop with mast cells, which sustain lymphoma cell growth ${ }^{12}$. We analyzed the $\mathrm{BM}$ of mice developing autoimmunity and characterized for the expansion of IFN- $\gamma+$ Teff ${ }^{16}$. Stromal cells of these mice expressed CD40, compared to untreated mice (Online Supplementary Figure 1A). These data suggested that the presence of inflammatory cytokines such as IFN- $\gamma$ could be relevant in increasing CD40 expression on BM-MSCs. Therefore we performed an in vitro analysis using primary human BM-MSCs and found that CD40 expression is induced by IFN- $\gamma$ paralleling the induction of IDO1, a well know immuneregulatory molecules (Online Supplementary Figure 1B). These data suggested that MSCderived CD40 could be relevant in the BM microenvironment when this microenvironment is perturbed toward the production of inflammatory cytokines.

Total body irradiation (TBI) is known to affect the BM immune microenvironment firstly inducing cytokines and then local immunosuppression to prevent the rejection of the donor bone marrow ${ }^{17}$. We evaluated whether CD40 expression, undetectable in the BMmicroenvironment in basal conditions, is induced by TBI. To this end BALB/c mice were lethally irradiated and sacrificed 4 or 7 days post-radiation to collect radiation-resistant BMMSCs ${ }^{18}$. Both RT-PCR (Figure 1A) on ex-vivo purified BM-MSCs (Online Supplementary Fig. 1C) and immunohistochemistry (IHC) (Figure 1B) showed that CD40 was overexpressed on total BM-MSCs at day 7 post-radiation compared to basal and day 4. The analysis of Tcell compartment showed the expansion of CD4+ cells upon irradiation both after 4 days and 7 days compared to non-irradiated mice (Figure 1C). Among CD4+ cells, we noticed that Teffs (CD4+Foxp3-) were increased in frequency compared to Tregs (CD4+Foxp3+) (Figure 1D). Moreover, the Teffs were activated to release TNF and IFN $\gamma$ (Figure 1E), which, tested in vitro, were able to induce strong CD40 up-regulation in ex-vivo isolated and in vitroexpanded BM-MSCs (Figure 1F) in comparison to other stimuli also released in the BM of conditioned mice (i.e., G-CSF, GM-CSF+IL-6, and IL-17) ${ }^{19}$ (Online Supplementary Figure 2A). 
To better characterize the BM-MSCs population expressing CD40 after TBI, we performed a multiparametric flow cytometry analysis. To define BM-MSCs we checked positivity for known surface MSC markers (CD44, CD29, Sca-1) and negativity for the lineage markers CD45, Ter119, CD34, CD117 and CD31. To our analysis we also added the CD73 marker, as it was recently attributed to radio-resistant and clonogenically-active BM-MSC ${ }^{20}$. Our analysis showed that within the gate of CD29+CD44+CD45-Ter119-CD31-CD34-CD117cells, lethal irradiation increased the frequency of Sca-1+ cells (Figure 1G). However, within both Sca-1+ and Sca-1- cells we found an increased frequency of CD73+ cells (Figure 1H). Paralleling CD73 we found that the frequency of CD40+ BM-MSCs was strongly increased after irradiation (Figure 1H). This suggests that CD40, as CD73, could be specifically induced and associated to radio-resistant BM-MSCs that could be either Sca-1+ or Sca-1-. Looking at the frequency of BM-MSCs co-expressing CD40 and CD73 within the gate of total BM-MSCs (CD29+CD44+CD45-Ter119-CD31-CD34-CD117-) we found an increased frequency of double positive $\mathrm{CD}^{+}{ }^{+} \mathrm{CD}^{-} 3^{-}$and $\mathrm{CD} 40+\mathrm{CD} 73+\mathrm{BM}-\mathrm{MSC}$ upon irradiation (Figure 1I). The representative gating strategy is shown in Figure 1J.

To test whether the gain of immune regulatory features in BM-MSCs occured at the expense of their differentiation program toward osteo-and adipo- lineages, we performed RT-PCR analyses on TNF and IFN- $\gamma$ stimulated cells. Osteoblast and adipocytes differentiation markers such as osterix, osteonectin, osteopontin, bglap, PPARg, were all down-modulated by TNF; osterix, Runx2, Sparc and Spp1 but not Bglap and PPARg were down-modulated by IFN- $\gamma$ (Online Supplementary 2B). The combination of TNF and IFN- $\gamma$ was additive in decreasing the expression of Sppl (Online Supplementary 2B). As control, the same MSCs showed increased CD40 expression, suggesting an inverse relationship between the expression of immune and differentiation programs.

\section{Altered B-cell lymphopoiesis in the BM is a characteristic of $\mathrm{Cd} 40-\mathrm{KO}$ recipient chimeric}

mice. To study the regulatory role of CD40 on BM-MSCs in vivo, we set up bone marrow transplant (BMT) experiments in which recipient mice (either Cd40-KO or WT, all CD90.2) were lethally irradiated and transplanted with HSCs from congeneic CD90.1 WT donors. Twenty-one days post-BMT we performed FACS on peripheral blood (PB), BM, and spleens of recipient mice. Compared to $\mathrm{WT}, C d 40-\mathrm{KO}$ recipients showed a prominent decrease in the frequency of PB B-cells and, to a lesser extent, of T-cells, but also an increased frequency of CD11b+ myeloid cells (Fig. 2A, Online Supplementary 3A). In line with our PB analysis, we 
observed a reduction of lymphoid cells in the $\mathrm{BM}$ of $\mathrm{Cd} 40-\mathrm{KO}$ recipients despite normal myelopoietic (CD11b cells) development (Figure 2B). Particularly, B-cell development was largely defective, as shown by the reduced B220+CD43+ and almost absent B220+CD43fractions (Figure 2B). B-cell development was arrested at the A and B fractions, which correspond to the pre-pro-B and early pro-B phases, respectively, with reduced development of the fraction of C'-C precursors (late pro-B and large pre-B) (Figure 2C; Online Supplementary 3B) ${ }^{15}$. Intriguingly, in the spleens of $C d 40-\mathrm{KO}$ recipients we noted an increased frequency of B220+CD43+ B-cell precursors that developed into mature B-cells of different types, thus giving rise to extramedullary B lymphopoiesis (Figure 2 D-E). However, the overall frequency of B220+ cells was significantly reduced in the spleen of $C d 40-\mathrm{KO}$ recipients (Figure 2F) with a significant impairment in the CD93+ immature fraction (particularly affected were transitional T1 cells, Figure 2G). Nevertheless, the presence of mature marginal zone B-cell and follicular B-cell (Figure 2H; Supplemental Figure 3C) suggested a compensatory role for splenic B-cell lymphopoiesis in response to dysfunctional BM B-lymphopoiesis. Notably, the defective B-cell lymphopoiesis in the $C d 40-\mathrm{KO}$ recipient chimeras was not due to a systemic anti-CD40 response as CD40+ B-cells were still present in the spleen and PB (Online Supplementary Figure 4A). The relevance of BM-MSC-derived CD40 in B cell development was also suggested by the close contact between CD40+ mesenchymal cells and B220+ cells in vivo as detected by confocal microscopy analysis for B220 and CD40 on BM biopsies (Figure 2I). We functionally proved this hypothesis by performing a BMT experiment where WT mice were lethally irradiated and reconstituted with Lin- cells in the presence of either WT or Cd40-KO BM-MSCs. BM-MSCs were also forced to express CXCR4 to promote BM homing. The presence of WT CXCR4+ BM-MSCs promoted a higher frequency of B220+ cells compared to mice that did not received MSCs (Figure 2J) at day 14 post-BMT. On the contrary, Cd40-KO BM-MSC negatively affected B cell development and 33\% of mice died (Figure 2J).

\section{CD40-deficiency in the BM stromal compartment creates a lymphopoietic niche unfit to} respond under stress condition. We next tested whether the lack of stromal CD40 expression might negatively impact on Treg development or generate a pro-inflammatory microenvironment, which in turn can inhibits B-cell development.

To this end, we analyzed BM T-cell status in $\mathrm{WT}>C d 40-\mathrm{KO}$ and $\mathrm{WT}>\mathrm{WT}$ chimeras, 4 weeks after BMT. Tregs were reduced in the BM of WT $>C d 40-\mathrm{KO}$ chimeras compared to the WT $>$ WT counterpart (Figure 3A), and IFN $\gamma$-producing Teff cells were significantly 
increased (Figure 3B, C). To gain further insight into the behavior of Tregs in the Cd40-KO recipients and their relationships with B-cell development, Tregs were co-infused with donor lin- cells (both B/c, H-2d) into either WT or Cd40-KO F1 recipients (both CxB6, H-2b/d) (Online Supplementary Figure 5A). Of note, Tregs accelerated the recovery of B-cells in BM of WT $>$ WT chimeras (Figure 3D), but not in $C d 40-\mathrm{KO}$ recipients. In line with this result, a reduced frequency of donor Tregs was observed in Cd40-KO compared to WT recipients (Figure 3E). Such reduction of donor Tregs was paralleled by decreased Foxp3 levels (Figure

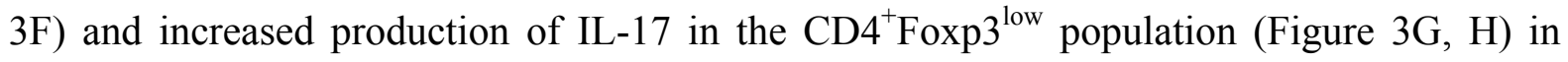
Cd40-KO recipients. This phenotype suggests a possible conversion of Tregs into Th17 cells ${ }^{21}$. In line, transplantation of WT donor cells into Cd40-KO recipients resulted in reduced OX40 expression on Tregs, a known marker of fitness ${ }^{22}$ (Online Supplementary Figure 5B). To try to explain this phenotype, we evaluated OX40L expression in $C d 40-\mathrm{KO}$ and wt BMMSCs. Indeed, previous data have shown that in the absence of an intact CD40/CD40L axis, OX40 triggering could worsen allograft rejection in cardiac transplant model, through the exacerbation of the Th1 and Th2 responses ${ }^{23}$. RT-PCR analysis performed onto ex-vivo stimulated BM-MSCs showed that Cd40-KO BM-MSCs had higher OX40L levels than their WT counterparts in response to TNF (Figure 3I). In line, BM IHC for OX40L showed an overall increase in its expression in Cd40-KO recipient mice (Figure 3J), and confocal microscopy analysis revealed co-staining with the nestin marker (Online Supplementary Figure 5C). These data could explain the observed impairment of Tregs in favor of Teffs in the $\mathrm{WT}>C d 40-\mathrm{KO}$ chimeras. In fact, the increased OX40L stimulation was previously shown to promote the conversion of Tregs to $\mathrm{Th} 17^{24}$. This is compatible with our earlier findings of increased production of IL-17 in Foxp $3^{\text {low }}$ cells of Cd40-KO recipient mice. Conversely, upon OX40L stimulation, Teff cells increase proliferation and acquire memory functions ${ }^{25,26}$. Overall, these data suggest that in the absence of CD40, BM-MSCs are unable to regulate donor Treg activity. Indeed, under certain inflammatory stimuli Treg may might lose FOXP3 expression and acquire the ability to produce Th cytokines ${ }^{27}$.

\section{Stromal CD40 expression is downregulated during BM manifestation of acute- GVHD}

disease. Allogeneic HSC transplantation is an effective treatment for many hematologic diseases, but one major treatment-associated complication is aGVHD. aGVHD patients often show impaired B-cell immunity as well as activated donor T-cell-mediated osteoblast destruction 2829 . Given the striking similarity of this phenotype with our findings in the BM of irradiated $C d 40-\mathrm{KO}$ mice, we set up experiments to evaluate whether the loss of CD40 
expression on BM-MSCs could be a common feature in BM experiencing an abnormal T-cell activation, such as during aGVHD pathogenesis. To model GVHD, we used an experimental model in which major histocompatibility complex (MHC)-mismatched BM cells and lymphocytes isolated from $\mathrm{C} 57 \mathrm{BL} / 6\left(\mathrm{H} 2^{\mathrm{b}}\right)$ donors were transplanted into $\mathrm{BALB} / \mathrm{c}\left(\mathrm{H} 2^{\mathrm{d}}\right)$ recipients. $2 \times 10^{6}$ T-depleted donor BM (TD-BM) cells were co-infused or not (control) with $5 \times 10^{5}$ donor CD4+CD25- (Teff) lymphocytes ${ }^{30}$. As a further control, a non-MHC mismatched BMT was also performed using BALB/c $\left(\mathrm{H}^{\mathrm{d}}\right)$ mice as both donor and recipient (Supplemental Fig. 6A). Under the allogeneic conditions, aGVHD manifested within 21 days as confirmed by changes in mice weight (Online Supplementary Figure 6B) and histological analysis of the liver, skin and lung, in accordance with a murine grading system (Online Supplementary Figure 6C-D) ${ }^{31}$. FACS analysis of BM cell suspensions isolated from these mice revealed impaired B-cell development associated with an increased production of IFN- $\gamma$ and TNF $\alpha$ by CD4 and CD8+ cells (Figure 4A). Notably, Treg frequency was significantly reduced in mice with aGVHD. Similar changes in the B-cell and Treg populations were also detected in PB samples, where a time-dependent loss of both cell types was observed (Figure 4B-C). These data suggest that Treg and B-cell frequencies could be used as early markers to identify patients who will likely develop aGVHD.

We next analyzed CD40 expression in the BM microenvironment during aGVHD. RT-PCR analysis showed loss of CD40 expression in freshly isolated BM-MSCs from aGVHD but not control chimeras (Figure 4D). IHC of the BM specimens confirmed CD40 loss during aGVHD (Figure 4E) and co-immunofluorescence analysis for nestin and CD40 confirmed the lack of CD40 on nestin+ stromal cells from aGVHD mice (Figure 4F). Notably, the lack of CD40 was coupled with increased OX40L on BM-MSCs (Figure 4G), which also showed increased production of NOS2 and IDO-I (Figure 4H). These data suggest that aGVHD development is associated with an altered immunoregulatory axis involving CD40 expression on BM-MSCs and Treg. The increased expression of Ido1 and Nos2 in BM-MSCs from aGVHD mice suggests that different mechanisms are in place to try to restore immune homeostasis. However, despite these mechanisms the outcome of BMT in CD40-deficient mice suggests that they cannot bypass the requirement for a functional CD40-CD40L axis.

Loss of MHC-I ${ }^{\text {high }}$ MSCs in BM from aGVHD mice. To try to explain the loss of CD40+ BM-MSCs we evaluated the expression of MHC-I, which is necessary for allo-recognition on BM-MSCs from lethally irradiated and aGVHD (TD-BM B6+Teff $>\mathrm{B} / \mathrm{c}$ ) vs controls (TD-BM 
$\mathrm{B} / \mathrm{c}+\mathrm{Teff}>\mathrm{B} / \mathrm{c}$ ) mice. Lethal irradiation increased the expression of MHC-I in Sca-1CD29+CD44+ BM-MSCs (Online Supplementary Figure 7). A similar trend was observed in Sca- $1+$ cells. Both Sca-1+ and Sca-1 ${ }^{-}$comprised CD40+ elements (not shown). The same analysis performed onto aGVHD vs controls mice, at 14 days post-transplantation, revealed a dramatic change in term of MHC-I+ cells composition in both the Sca-1+ and Sca-1fraction. Indeed, in both populations we found an almost complete loss of MHC-I ${ }^{\text {high }} \mathrm{CD} 40+$, and $\mathrm{MHC}^{\text {high }} \mathrm{CD} 40$ - subsets, notably leaving intact those expressing lower level of MHC-I (Figure 5A-C).

The absence of CD40 specifically worsen the BM manifestation of aGVHD. To assess the relevance of CD40 in the context of aGVHD, Cd40-KO and WT mice were transplanted with MHC-mismatched TD-BM cells co-infused or not with donor CD4+CD25- Teff. We found that transplantation of $C d 40-\mathrm{KO}$ mice with a MHC-mismatched BM was sufficient to promote an excessive production of inflammatory cytokines in the BM even in the absence of donor Teff (Figure 6A), so much that recipient mice were sacrificed at day 14 instead of day 21 because of their suffering conditions (at day $12,15 \%$ of $C d 40-\mathrm{KO}$ recipients were died). In these mice, FACS analysis of BM cells showed a significant increase in the frequency of $\mathrm{CD} 3+\mathrm{T}$ cells indicating that the $C d 40-\mathrm{KO}$ microenvironment favors the proliferation and pro-inflammatory activation of radioresistant host $\mathrm{T}$ cells (but also donor $\mathrm{T}$ cells, when coinfused). Indeed, we found high production of IFN- $\gamma$ and TNF by CD4-T cells along with decreased frequency of B220+ cells (Figure 6A). Notably, the reduced frequency of donor TD-BM cells in Cd40-KO compared to WT recipients (not shown), suggests that the Cd40$K O$ microenvironment might favour the generation of a graft vs donor response.

Histological and IHC analysis showed a reduced BM cellularity and increased expression of OX40L on mesenchymal elements of Cd40-KO recipients (Figure 6B). Notably, the exacerbated inflammatory condition due to CD40 deficiency in radio-resistant cells, both in presence and absence of donor Teff, was restricted to the BM environment, as the histology of infiltrating lymphocytes in peripheral organs (liver and skin) was not different between WT and Cd40-KO recipients (Online Supplementary Figure 8).

Association between CD40 and PAX5 loss in aGvHD patients BOM. To assess the relevance of our findings in human disease, we examined BM biopsies from 12 allo-HSCT patients, seven of which developed aGVHD (grade I-III). Patient's characteristics are 
included in Supplemental Table 1. BM Paraffin sections were stained with Ab to CD40 and to PAX5, an early B-cell transcription factor expressed since the early stages of B-cell lymphopoiesis. A blinded analysis of these cases showed that CD40 was either not expressed, or expressed on scattered elements with myeloid morphology, or in spindle- or stellateshaped stromal cells in other different cases. The mesenchymal nature of the spindle- or stellate-shaped cells was confirmed by double-marker immunofluorescence analysis for CD40 and CD146 (Figure 7A). Pax5 expression ranged from less than $1 \%$ to nearly $20 \%$ of the hematopoietic cellularity and positively correlated with CD40 expression on mesenchymal cells. B-cell frequencies were lower in cases of non-stromal or negative expression of CD40. The last condition characterized GVHD patients (Figure 7B and Online Supplementary Table 1) in full agreement with the described mouse data. The analysis of the expression and localization of granzyme-B in BOM from GVHD patients showed that patients that had a strong reduction in PAX5+ cells had granzyme $\mathrm{B}+$ granules localized onto CD146+ BM-MSCs cells (Figure 7C), suggesting that Teff cells are actively killing BMMSCs. 


\section{Discussion}

The bone marrow (BM) is a peculiar lymphoid organ. Mature and memory T cells relocate in the BM parenchyma, which is a potential source of effector T cells ${ }^{32}$. This could, at least in part, explain why the BM is the organ with the highest frequency of Treg compared to other secondary lymphoid organs, such as spleen and $\mathrm{LN}^{33}$. Furthermore, the strong detrimental effect of uncontrolled cytokine production over HSC homeostasis and differentiation might explain the need of high Treg activity to keep effector T cells silent ${ }^{1}$. This is particularly evident for B-cells. In fact, the initial phases of B-cell development is influenced by the BM immune environment. In fact an excessive IFN- $\gamma$ production ${ }^{5}$ or changes in Treg composition ${ }^{3}$ has been shown to negatively affect B-cell development.

Here, we provide evidence that the immune regulatory activity of BM-MSCs over Treg and Teff, which is generally ascribed to soluble molecules (i.e. IDO, NOS-2), relies on the expression of CD40, a cell surface receptor belonging to the TNF-R family which function is critical for the antigen presenting cells activity ${ }^{34}$. Starting from our previous finding of CD40 up-regulation in CD146+ mesenchymal cells of SMZL patients, we show here that CD40 expression in the BM microenvironment is induced under inflammatory conditions, such as those associated with lethal irradiation. Modelling BMT in mice, we show that the postradiation inflammatory response, guided by IFN- $\gamma$ and TNF, is sufficient to induce CD40. BM-MSCs and Foxp3+ Treg cells closely interact to confer immune privilege to $\mathrm{BM}^{35}$. We showed that the prompt up-regulation of CD40 in radio-resistant BM-MSCs during TBI, is required to grant Treg functions. Accordingly, the absence of CD40 on stromal elements reduces Tregs in favor of Teff, possibly via conversion to Th17 cells.

This condition was associated to defective B-cell development in $\mathrm{Cd} 40$-KO mice, mirroring the prolonged B-cell dysfunction, the increased T-cell infiltration and the disrupted BM niche that characterize human aGVHD ${ }^{29}$. Indeed, BM biopsies from aGVHD patients reveal lack of PAX5 expression, a marker that identifies early B-cells, along with the loss of stromal CD40 expression.

Mechanistically, we found that the absence of CD40 on stromal cells was associated to higher level of OX40L expression, a feature underestimated in BM-MSCs. Our data suggest that in the absence of CD40/CD40L, the OX40/OX40L axis might prevail. In line, stimulation of OX40 overrides cardiac allograft acceptance induced by disrupting CD40-CD40L interaction

${ }^{23}$. In the BM this might favor Teff proliferation at the expense of Treg, thus creating a pro- 
inflammatory environment that in case of MHC-mismatch, might favor the development of aGVHD.

The loss of CD40+ BM-MSCs in aGVHD, might be due to their high MHC-I expression. Indeed, modeling GVHD in mice we found a time-dependent loss of MHC-I-high MSC subset, which comprises CD40+ cells. This finding opens two possible scenarios: the first one is the existence of a MHC-driven allo-recognition and therefore elimination of $\mathrm{MHC}-\mathrm{I}^{+}$ BM-MSCs, and the second one is the possible down-modulation of MHC-I molecules on BM-MSCs, to prevent allo-recognition. BM-biopsies from aGVHD patients showing the disruption of osteoblasts of the BM niche ${ }^{29}$ together with our data showing the colocalization of granzyme-B granules with nestin+ BM-MSCs in the same aGVHD patients strongly support the first hypothesis.

Furthermore, analyzing the compartment of radio-resistant BM-MSCs we show that lethal irradiation increases the frequency of CD40+ BM-MSCs and that a subset of these cells are CD73+. Indeed, CD73 is an ectoenzyme involved in the production of adenosine, a potent immune suppressive molecule, also affecting Treg ${ }^{36}$. This might explain why the loss of CD40+ BM-MSCs subset during GVHD results in exacerbated inflammatory response.

Different clinical trials testing the efficacy of BM-MSCs to prevent or treat GVHD are running ${ }^{37}$. Most of them utilize allo-MSCs from third-party donors. Results from these trials show no collateral side effects and a good overall response rate in chronic GVHD patients. In aGVHD patients no significant differences have been shown among MSCs vs no MSCs groups, although encouraging results have been reported in one controlled clinical trial with 40 patients in which a complete or partial response was observed in 15 and 1 patient, respectively. Several clinical trials are still on going and will provide additional data to evaluate the efficacy of allo-MSC administration. Nevertheless, some key factors that can influence MSCs activity have been identified, such as the route of administration and the source of allo-MSCs. On this regards our data provide new insight suggesting that although the total MSCs population is potentially able to provide immune suppression, only the specific subset of BM-MSCs expressing CD40 could be the most effective for the generation of BM tolerance. However, their tolerogenic effect could be reduced because of their high expression of MHC-I molecules, which may lead to their elimination.

Finally, the study shows that the gaining of regulatory functions along with CD40 upregulation, in MSCs is associated with the down-regulation of their speciation markers (Bglap, Osterix, Osteopontin) in favor of immune regulatory feauture. This is either 
explained by a more undifferentiated/progenitor subset of BM-MSCs exerting these functions, or by a switch from an architectural/supportive hematopoietic niche function of BM-MSCs to an immunoregulatory activity when excessive inflammatory conditions are sensed. Additionally, here we described that high expression of CD40 is on Sca-1+, a marker that identifies stromal progenitors able to generate both osteogenic and stromal cells and able to provide a supportive environment for hematopoiesis ${ }^{38}$. It has been reported that Sca-1positive cells have higher colony forming ability and show enhanced proliferation compared with Sca-1-negative cells ${ }^{39}$. Overall, all these pieces of evidence support the possibility that the subset of BM-MSCs mostly endowed with immune regulatory function could be the mostly undifferentiated.

In conclusion, we provide evidence that stromal CD40 expression is up-regulated in BM-MSCs after irradiation as a step necessary to restore $\mathrm{T}$ cell homeostasis during HSCT. CD40-deficiency in BM stromal cells skews BM $\mathrm{T}$ cell activation towards the persistent production of inflammatory molecules that, in turn, impair normal B cell development. These CD40-dependent changes are pathogenetically relevant in human aGVHD. Based on our data, CD40 appears to be a key molecule conferring tolerogenic properties to BM niches. 


\section{Acknowledgments}

The authors thank the Conventional and Confocal Microscopy Facility for confocal images acquisition. This work was supported by the Italian Ministry of Health (GR-2013-02355637 to S. Sangaletti); and Associazione Italiana per la Ricerca sul Cancro (Investigator Grant number 22204 to Sabina Sangaletti and 10137 to M.P. Colombo). Barbara Bassani is funded by the FIRC-AIRC (Fondazione Italiana per la Ricerca sul Cancro) fellowship "Guglielmina Lucatello e Gino Mazzega". The authors also thank E. Grande for administrative support.

\section{Author contributions}

S.S and M.P.C. designed research; B.B. A.P., A.G. P.P. L.B. B.C. M.C. performed the experiments, S.S., I.N. K.J. I.A. provided human biopsies and analysed clinical parameters, C.C. C.T. A.C. analyzed the data. S.S. N.B., C.T. and M.P.C wrote the manuscript.

\section{Additional information}

Supplemental Information accompanies this paper.

Competing interests: The authors declare no competing interests. 
references

1. Hirata Y, Furuhashi K, Ishii H, et al. CD150(high) Bone Marrow Tregs Maintain Hematopoietic Stem Cell Quiescence and Immune Privilege via Adenosine. Cell Stem Cell. 2018;22(3):445-453 e445.

2. Danby RD, Zhang W, Medd P, et al. High proportions of regulatory T cells in PBSC grafts predict improved survival after allogeneic haematopoietic SCT. Bone Marrow Transplant. 2016;51(1):110-118.

3. Pierini A, Nishikii H, Baker J, et al. Foxp3(+) regulatory $\mathrm{T}$ cells maintain the bone marrow microenvironment for B cell lymphopoiesis. Nat Commun. 2017;8:15068.

4. Kim S, Park K, Choi J, et al. Foxp3+ regulatory T cells ensure B lymphopoiesis by inhibiting the granulopoietic activity of effector $\mathrm{T}$ cells in mouse bone marrow. Eur $J$ Immunol. 2015;45(1):167-179.

5. Baratono SR, Chu N, Richman LP, Behrens EM. Toll-like receptor 9 and interferongamma receptor signaling suppress the B-cell fate of uncommitted progenitors in mice. Eur $J$ Immunol. 2015;45(5):1313-1325.

6. Maloy KJ, Powrie F. Fueling regulation: IL-2 keeps CD4+ Treg cells fit. Nat Immunol. 2005;6(11):1071-1072.

7. Guiducci C, Valzasina B, Dislich H, Colombo MP. CD40/CD40L interaction regulates $\mathrm{CD} 4+\mathrm{CD} 25+\mathrm{T}$ reg homeostasis through dendritic cell-produced IL-2. Eur $J$ Immunol. 2005;35(2):557-567.

8. Ehninger A, Trumpp A. The bone marrow stem cell niche grows up: mesenchymal stem cells and macrophages move in. J Exp Med. 2011;208(3):421-428.

9. Uccelli A, Pistoia V, Moretta L. Mesenchymal stem cells: a new strategy for immunosuppression? Trends Immunol. 2007;28(5):219-226.

10. Le Blanc K, Rasmusson I, Sundberg B, et al. Treatment of severe acute graft-versushost disease with third party haploidentical mesenchymal stem cells. Lancet. 2004;363(9419):1439-1441.

11. Meisel R, Zibert A, Laryea M, Gobel U, Daubener W, Dilloo D. Human bone marrow stromal cells inhibit allogeneic T-cell responses by indoleamine 2,3-dioxygenase-mediated tryptophan degradation. Blood. 2004;103(12):4619-4621.

12. Franco G, Guarnotta C, Frossi B, et al. Bone marrow stroma CD40 expression correlates with inflammatory mast cell infiltration and disease progression in splenic marginal zone lymphoma. Blood. 2014;123(12):1836-1849.

13. Tripodo C, Sangaletti S, Guarnotta C, et al. Stromal SPARC contributes to the detrimental fibrotic changes associated with myeloproliferation whereas its deficiency favors myeloid cell expansion. Blood. 2012;120(17):3541-3554.

14. Sangaletti S, Tripodo C, Portararo P, et al. Stromal niche communalities underscore the contribution of the matricellular protein SPARC to B-cell development and lymphoid malignancies. Oncoimmunology. 2014;3:e28989.

15. Hardy RR, Hayakawa K. B cell development pathways. Annu Rev Immunol. 2001;19:595-621.

16. Tripodo C, Burocchi A, Piccaluga PP, et al. Persistent Immune Stimulation Exacerbates Genetically Driven Myeloproliferative Disorders via Stromal Remodeling. Cancer Res. 2017;77(13):3685-3699.

17. Paix A, Antoni D, Waissi W, et al. Total body irradiation in allogeneic bone marrow transplantation conditioning regimens: A review. Crit Rev Oncol Hematol. 2018;123:138148 . 
18. Nicolay NH, Lopez Perez R, Saffrich R, Huber PE. Radio-resistant mesenchymal stem cells: mechanisms of resistance and potential implications for the clinic. Oncotarget. 2015;6(23):19366-19380.

19. Melenhorst JJ, Tian X, Xu D, et al. Cytopenia and leukocyte recovery shape cytokine fluctuations after myeloablative allogeneic hematopoietic stem cell transplantation. Haematologica. 2012;97(6):867-873.

20. Severe N, Karabacak NM, Gustafsson K, et al. Stress-Induced Changes in Bone Marrow Stromal Cell Populations Revealed through Single-Cell Protein Expression Mapping. Cell Stem Cell. 2019;25(4):570-583 e577.

21. Komatsu N, Okamoto K, Sawa S, et al. Pathogenic conversion of Foxp3+ T cells into TH17 cells in autoimmune arthritis. Nat Med. 2014;20(1):62-68.

22. Piconese S, Pittoni $\mathrm{P}$, Burocchi A, et al. A non-redundant role for OX40 in the competitive fitness of Treg in response to IL-2. Eur J Immunol. 2010;40(10):2902-2913.

23. Burrell BE, Lu G, Li XC, Bishop DK. OX40 costimulation prevents allograft acceptance induced by CD40-CD40L blockade. J Immunol. 2009;182(1):379-390.

24. Vu MD, Xiao X, Gao W, et al. OX40 costimulation turns off Foxp3+ Tregs. Blood. 2007;110(7):2501-2510.

25. Redmond WL, Ruby CE, Weinberg AD. The role of OX40-mediated co-stimulation in T-cell activation and survival. Crit Rev Immunol. 2009;29(3):187-201.

26. Piconese S, Valzasina B, Colombo MP. OX40 triggering blocks suppression by regulatory T cells and facilitates tumor rejection. J Exp Med. 2008;205(4):825-839.

27. Li Z, Li D, Tsun A, Li B. FOXP3+ regulatory T cells and their functional regulation. Cell Mol Immunol. 2015;12(5):558-565.

28. Shono Y, Ueha S, Wang Y, et al. Bone marrow graft-versus-host disease: early destruction of hematopoietic niche after MHC-mismatched hematopoietic stem cell transplantation. Blood. 2010;115(26):5401-5411.

29. Mensen A, Johrens K, Anagnostopoulos I, et al. Bone marrow T-cell infiltration during acute GVHD is associated with delayed B-cell recovery and function after HSCT. Blood. 2014;124(6):963-972.

30. Schroeder MA, DiPersio JF. Mouse models of graft-versus-host disease: advances and limitations. Dis Model Mech. 2011;4(3):318-333.

31. Naserian S, Leclerc M, Thiolat A, et al. Simple, Reproducible, and Efficient Clinical Grading System for Murine Models of Acute Graft-versus-Host Disease. Front Immunol. 2018;9:10.

32. Schirrmacher V, Feuerer M, Fournier P, Ahlert T, Umansky V, Beckhove P. T-cell priming in bone marrow: the potential for long-lasting protective anti-tumor immunity. Trends Mol Med. 2003;9(12):526-534.

33. Zou L, Barnett B, Safah H, et al. Bone marrow is a reservoir for CD4+CD25+ regulatory $\mathrm{T}$ cells that traffic through CXCL12/CXCR4 signals. Cancer Res. 2004;64(22):8451-8455.

34. Elgueta R, Benson MJ, de Vries VC, Wasiuk A, Guo Y, Noelle RJ. Molecular mechanism and function of CD40/CD40L engagement in the immune system. Immunol Rev. 2009;229(1):152-172.

35. Fujisaki $\mathrm{J}, \mathrm{Wu} \mathrm{J}$, Carlson $\mathrm{AL}$, et al. In vivo imaging of Treg cells providing immune privilege to the haematopoietic stem-cell niche. Nature. 2011;474(7350):216-219.

36. Allard B, Longhi MS, Robson SC, Stagg J. The ectonucleotidases CD39 and CD73: Novel checkpoint inhibitor targets. Immunol Rev. 2017;276(1):121-144.

37. Fisher SA, Cutler A, Doree C, et al. Mesenchymal stromal cells as treatment or prophylaxis for acute or chronic graft-versus-host disease in haematopoietic stem cell 
transplant (HSCT) recipients with a haematological condition. Cochrane Database Syst Rev. 2019; 1:CD009768.

38. Hu X, Garcia M, Weng L, et al. Identification of a common mesenchymal stromal progenitor for the adult haematopoietic niche. Nat Commun. 2016;7:13095.

39. Baustian C, Hanley S, Ceredig R. Isolation, selection and culture methods to enhance clonogenicity of mouse bone marrow derived mesenchymal stromal cell precursors. Stem Cell Res Ther. 2015;6:151. 


\section{Figure Legends}

Figure 1. Expression of CD40 in the BM microenvironment. A. RT-PCR showing the expression of CD40 on primary BM-MSCs (not expanded in vitro) purified from BM at day 4 and 7 post-radiation and compared to basal. B. Representative image of CD40 IHC staining in the femurs and tibias of WT mice harvested 7 days post-irradiation. C. FACS analysis showing the frequency of CD4+ and CD8 T-cells in lethally irradiated (not reconstituted) mice ( $\mathrm{n}=5$ per group). ${ }^{* *} \mathrm{p}<0.005,{ }^{* * *} \mathrm{p}<0.001$, compared by unpaired $t$ test. D. Frequency of Teffs (CD25-Foxp3-) and Tregs (CD25+Foxp3+) in irradiated mice (CD4 gate) ( $\mathrm{n}=5$ per group). ${ }^{* *} \mathrm{p}<0.005,{ }^{* * *} \mathrm{p}<0.001$, compared by unpaired $t$ test. E. FACS analysis showing the frequency of Teff producing IFN $\gamma$ and TNF (CD4+Foxp3-gate) ( $\mathrm{n}=5$ per group). ${ }^{* *} \mathrm{p}<0.005,{ }^{* * *} \mathrm{p}<0.001$, compared by unpaired $t$ test. F. Real-time PCR analysis for $C d 40$ performed on total RNA isolated from in vitro expanded BM-MSCs stimulated $24 \mathrm{~h}$ with $10 \mathrm{ng} / \mathrm{ml}$ IFN $\gamma$ and TNF or their combination $\left(\mathrm{n}=5\right.$ per group). ${ }^{* *} \mathrm{p}<0.005,{ }^{* * *} \mathrm{p}<$ 0.001 , compared by unpaired $t$ test. G. Cumulative FACS analysis showing the frequency of Sca-1+ and Sca-1- BM-MSCs (CD29+CD44+CD45-Ter119-CD31-CD117-CD34- gate) in irradiated mice. H. Cumulative FACS analysis showing the frequency of CD73+ and CD40+ BM-MSCs within the Sca-1+ and Sca-1-gate. I. Cumulative FACS analysis showing the frequency of CD40+CD73-, CD40+CD73+ and CD40-CD73+ BM-MSCs in irradiated (RAD) vs non-irradiated mice (CTRL). $\mathrm{N}=8$ for controls and $\mathrm{n}=10$ for irradiated mice; **p < $0.005, * * * \mathrm{p}<0.001, * * * \mathrm{p}<0.0001$, multiple comparisons using a one-way ANOVA. J. Representative gating strategy for CD73 positive, CD40 positive and CD40/CD73 double positive MSCs is shown.

Figure 2. Analysis of B-cell development in WT $>W T$ and $W T>C d 40-K O$ BM chimeras. A Cumulative data for the PB FACS analysis showing the frequencies of $\mathrm{B} 220+, \mathrm{CD} 3+$, $\mathrm{CD} 11 \mathrm{~b}+$ cells as well as the $\mathrm{B} 220 / \mathrm{CD} 3$ ratio in the $\mathrm{PB}$ of $\mathrm{WT}>C d 40-\mathrm{KO}$ compared to WT $>$ WT BM chimeras. Representative plots and gating strategy are shown in Supplemental Figure 2a B. Cumulative data for FACS analysis of the BM showing the overall decrease in $\mathrm{B} 220+\mathrm{CD} 43+$ and $\mathrm{B} 220+\mathrm{CD} 43-\mathrm{B}-$ cell subsets in $\mathrm{Cd} 40-\mathrm{KO}$ recipients along with an increase in the frequency of $\mathrm{CD} 11 \mathrm{~b}+$ myeloid cells $* \mathrm{p}<0.05 \quad$ C. Cumulative data showing the frequency of pre-pro-B and early pro-B precursors (A and B fractions), and of late pro-B and large pre-B precursors $\left(C^{\prime}-C\right)$ in chimeric mice; D. Cumulative data showing the frequency of $\mathrm{B} 220+\mathrm{CD} 43+\mathrm{B}$-cell precursors in the spleens of $\mathrm{Cd} 40-\mathrm{KO}$ recipient mice $(\mathrm{n}=6$ per 
group). E. Representative dot plots showing that B220+CD43+ cells are almost absent in the spleen of WT but not Cd40-KO recipients. F. Frequency of B220+ B-cells is lower in the spleen of chimeric mice than in WT mice. G. Frequency of B220+CD93+ and B220+CD93B-cells in the spleen of chimeric mice. Splenic immature B220+CD93+ B-cell were divided into transitional T1, T2, and T3 cells based on their expression of CD23 and $\operatorname{IgM}(\mathrm{T} 1=\operatorname{IgM}$ $\left.+\mathrm{CD} 23-, \mathrm{T} 2=\operatorname{IgM}+\mathrm{CD} 23+, \mathrm{T} 3=\operatorname{IgM}{ }^{\text {low }} \mathrm{CD} 23+\right)$. H. Frequency of MZB and FoB within the gate of B220+CD93- cells is also highlighted. The relative gating strategy is shown in Supplemental Figure $2 * \mathrm{p}<0.05$, compared using Student's $t$ test for all analyses. I Confocal microscopy analysis for CD40 (green) and B220 (red) expression showing the close contact between B220+ and CD40+elements in WT $>$ WT BM chimeras. J. Dot plots showing B cell reconstitution in WT mice transplanted with Lin- cells co-infused with WT or Cd40-KO BMMSCs transduced or not with CXCR4 (n=3/group).

Figure 3. Characterization of $\mathrm{T}$-cell status in the $C d 40-\mathrm{KO}$ recipient BM chimeras. A. Treg frequency in the BM of WT $>\mathrm{WT}$ and $\mathrm{WT}>C d 40-\mathrm{KO}$ BM chimeras ( $\mathrm{n}=6$ per group). $* * * \mathrm{p}<0.001$, compared using Student's $t$ test. B. Cumulative data and C. representative dot gating strategy showing the production of IFN $\gamma$ by CD4+Foxp3- Teffs $s$ in the BM of $\mathrm{WT}>\mathrm{WT}$ and $\mathrm{WT}>C d 40-\mathrm{KO}$ BM chimeras ( $\mathrm{n}=6$ per group). ${ }^{*} \mathrm{p}<0.05$, compared by Student's $t$ test. D. Frequency of B220+ cells in the WT $>$ WT and WT $>C d 40-\mathrm{KO}$ BM chimeras after lethally irradiating CxB6F1 WT or Cd40-KO mice with lin- cells and spleenderived Tregs from donor BALB/c mice ( $n=5$ per group). ${ }^{*} p<0.05$, compared by Student's $t$ test. Please see Supplemental Figure 4 a for a schematic representation of this experiment. E. Frequency of donor $\left(\mathrm{H}-2 \mathrm{Kd}^{+} \mathrm{Kb}^{-}\right) \mathrm{CD} 4+\mathrm{Foxp} 3+$ Tregs in the WT $>\mathrm{WT}$ and $\mathrm{WT}>C d 40-\mathrm{KO}$ BM chimeras reconstituted with lin- and Tregs ( $\mathrm{n}=5$ per group). ${ }^{*} \mathrm{p}<0.05$, compared by Student's $t$ test. F. Cumulative data showing the MFI of Foxp3 on Treg cells. G. IL-17 production by Foxp $3^{\text {low }}$ Tregs in Cd40-KO recipients ( $n=5$ per group). ${ }^{* *}$ p $<0.005$, compared by Student's t test. H. Representative gating strategy for Foxp3 and IL-17 analysis in the different BM chimeras. I. Quantitative RT-PCR analysis of $O x 40 l$ gene expression in WT and Cd40-KO BM-MSCs treated in vitro with IFN- $\gamma$, TNF, or their combination ( $\mathrm{n}=5$ per group). $* * * * p<0.0001$, multiple comparisons using a one-way ANOVA. J. Representative images of OX40L staining in BM sections of the WT $>$ WT and WT $>C d 40$ KO BM chimeras. Mesenchymal-like elements (arrows) are shown in greater detail in Supplemental Fig. 4c. 
Figure 4 Downregulation of CD40 expression in the BM-MSCs of aGVHD mice. A. Frequency of B220+ B-cells, CD4+Foxp3- Teffs releasing IFN $\gamma$ and TNF, CD8+ T-cells releasing IFN $\gamma$ and TNF and CD4+Foxp3+ Tregs in the aGVHD mice compared to control animals. ${ }^{*} \mathrm{p}<0.05,{ }^{* *} \mathrm{p}<0.005, * * * \mathrm{p}<0.001$, compared by Student's $t$ test. PB FACS analysis showing changes in the frequency of B. B-cells and C. Tregs at days 7, 14, and 21 post-allogeneic BMT compared to controls ( $\mathrm{n}=5$ per group). ${ }^{* * *} \mathrm{p}<0.001$, compared by Student's $t$ test. D. qPCR analysis of $C d 40$ expression in BM-MSCs isolated from aGVHD and control mice. ${ }^{* *} \mathrm{p}<0.005,{ }^{* * *} \mathrm{p}<0.001$, compared by Student's $t$ test. E. Representative CD40 IHC staining in aGVHD mice (TD-BM B6 + Teff $>$ BALB/c) compared to controls (TD-BM BALB/c + Teff $>$ BALB/c). F. Representative co-immunofluorescence showing CD40 (green) and nestin (red) levels in aGVHD mice (TD-BM B6 + Teff $>$ BALB/c) compared to controls (TD-BM BALB/c + Teff $>$ BALB/c). G. Representative images of OX40L IHC staining in BM sections from allogeneic-transplanted animals (TD-BM + Teff B6 $>$ B/c) or controls (TD-BM BALB/c + Teff $>$ BALB/c). H. Quantitative RT-PCR analysis of Ido1, and Nos 2 expression in BM-MSCs isolated from allogeneic-transplanted animals (TD$\mathrm{BM}+$ Teff B6>B/c) and controls (TD-BM BALB/c + Teff $>$ BALB/c).

Figure 5 MHC-I ${ }^{\text {high }}$ MSCs are specifically eliminated in aGVHD mice. A. Frequency of $\mathrm{MHC}^{\text {high }} \mathrm{CD} 40+, \mathrm{MHC}^{\text {high }} \mathrm{CD} 40-$ and of $\mathrm{MHC}^{\text {neg }} \mathrm{CD} 40+\mathrm{BM}-\mathrm{MSC}$ in the Sca-1+ gate and $\mathbf{B}$. Sca- $1^{\text {neg }}$ gate of Lin-CD44+CD29+ BM-MSCs in aGVHD vs controls mice. The analysis was performed at 14 days post-transplantation. ${ }^{*} \mathrm{p}<0.05, * * \mathrm{p}<0.005, * * * \mathrm{p}<0.001$, compared by Student's $t$ test, ****p $<0.0001$, compared by Student's $t$ test. C. Representative gating strategy for the above analysis.

Figure 6 OX40L expression is increased in the BM of aGVHD mice. A. Frequency of $\mathrm{CD} 3, \mathrm{IFN} \gamma+$ and TNF+ Teff and B220+ cells in Cd40-KO and WT mice receiving an MHCmismatched BM. ${ }^{*} \mathrm{p}<0.05,{ }^{*} \mathrm{p}<0.005,{ }^{* * *} \mathrm{p}<0.001$, compared by Student's $t$ test. B. Representative H\&E and OX40L IHC images of BM sections from aGVHD (TD-BM + Teff B6 $>$ B/c) $v s$ controls (TD-BM BALB/c + Teff $>$ BALB/c) chimeras.

Figure 7. Lack of CD40+ BM-MSCs in BOM from aGVHD patients. A. Representative double-marker immunofluorescence analysis for CD40 (red) and CD146 (green), showing 
CD40 expression shared by CD146+ mesenchymal elements (red arrows) or confined to CD146- hematopoietic cells (white arrows). B. Pax5 IHC showing a variable expression of Pax5 with the highest fractions of Pax5+ cells observed in cases in which CD40 was expressed also in the mesenchymal cells. Patient's characteristics and quantitative IHC data are included in Supplemental Table 1. 
Figure 1

A

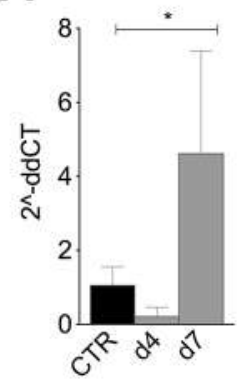

D

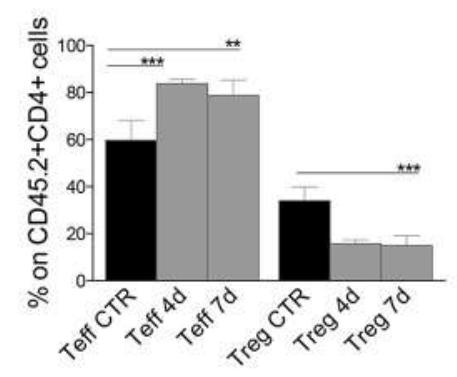

B

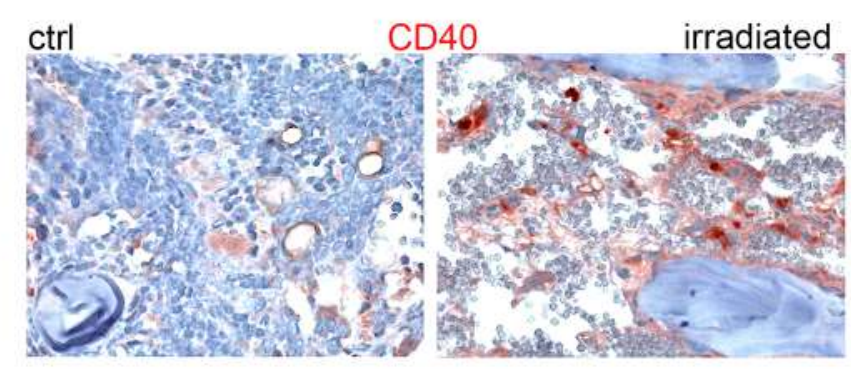

E

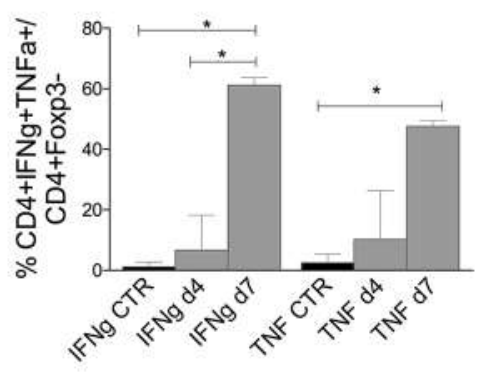

C

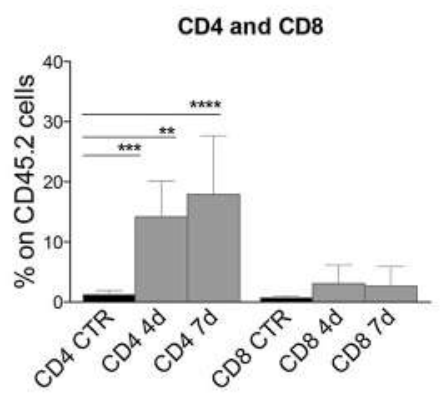

$\mathbf{F}$

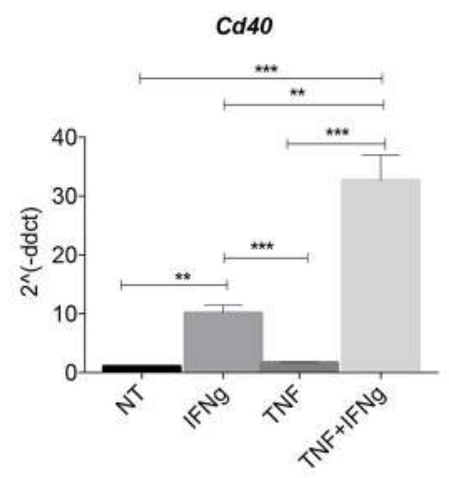

G

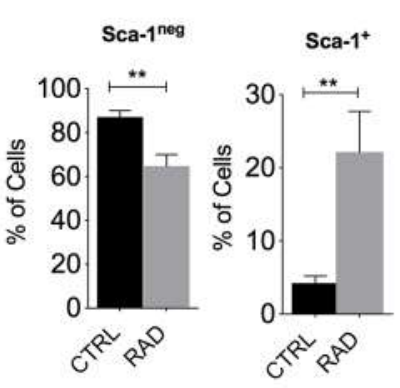

H

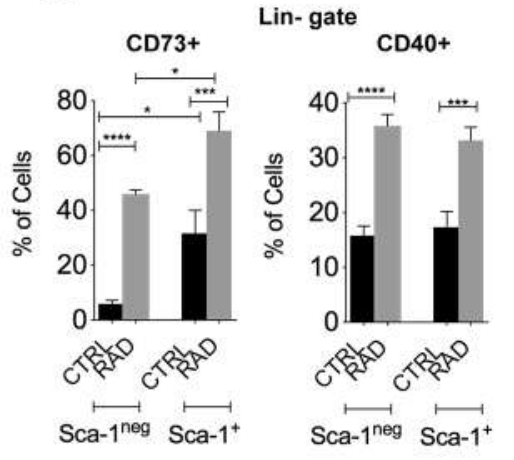

\section{I}

CD40+CD73-

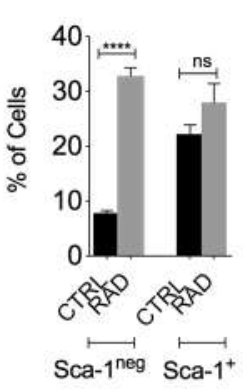

CD44+CD29+ gate CD40+CD73+

CD40-CD73+

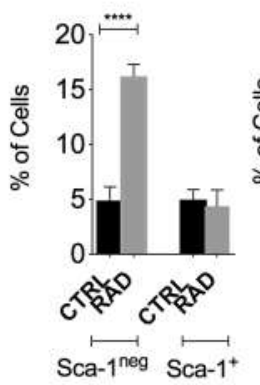

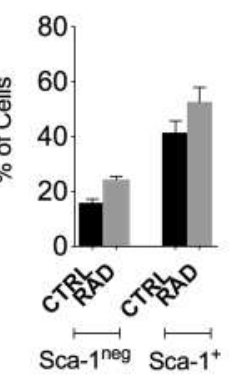

J
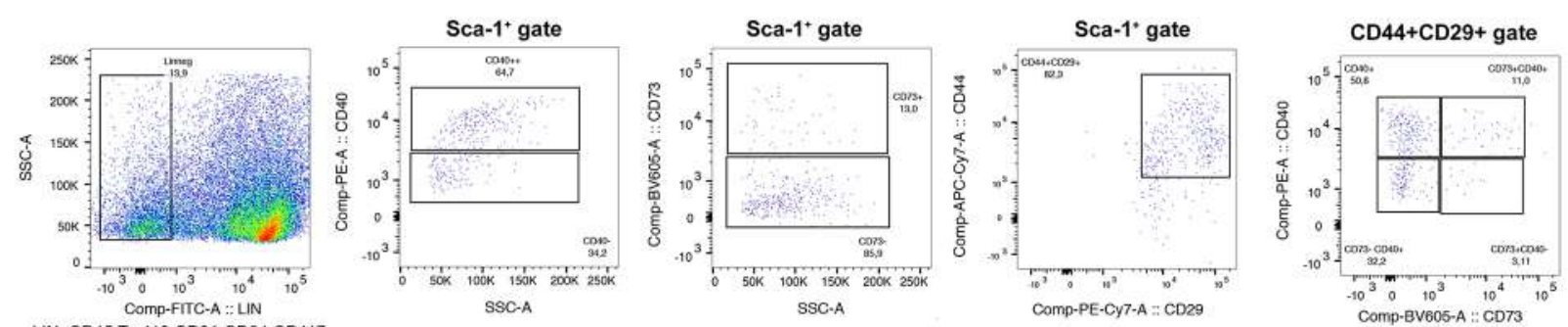

LIN: CD45 Ter119 CD31 CD34 CD117
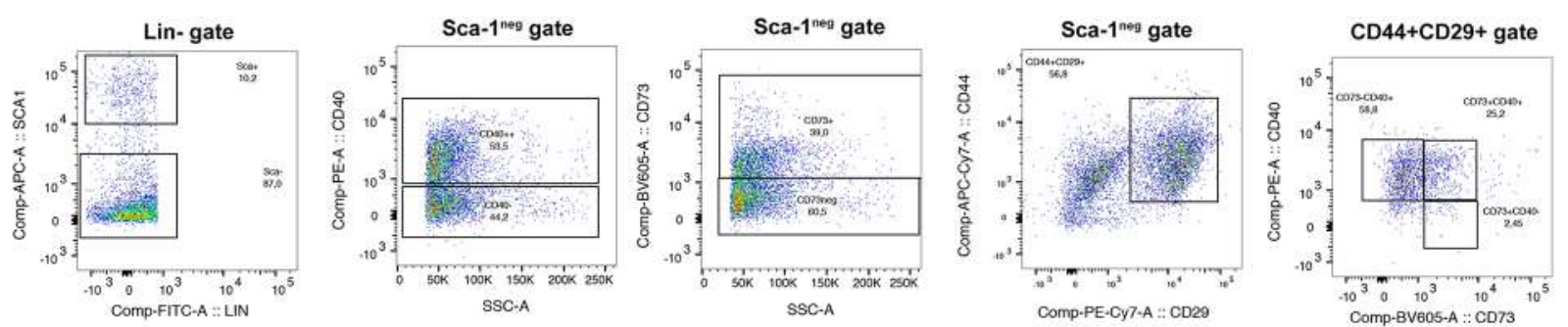

LIN : CD45 Ter119 CD31 CD34 CD117 
Figure 2

$\mathbf{A}$

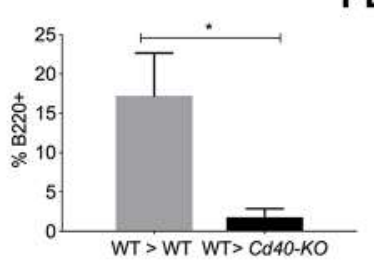

PB

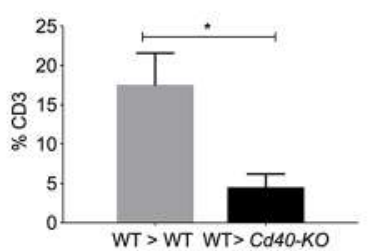

C

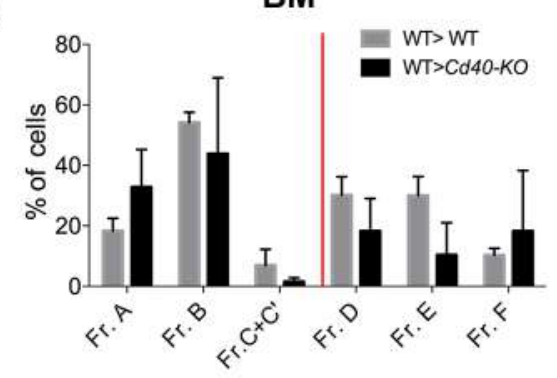

B220+CD43+ gate

F

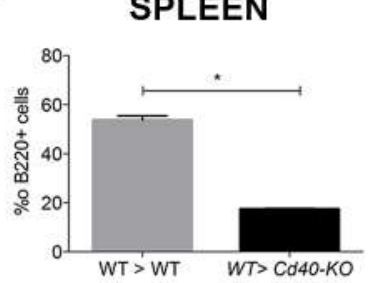

$\mathbf{G}$
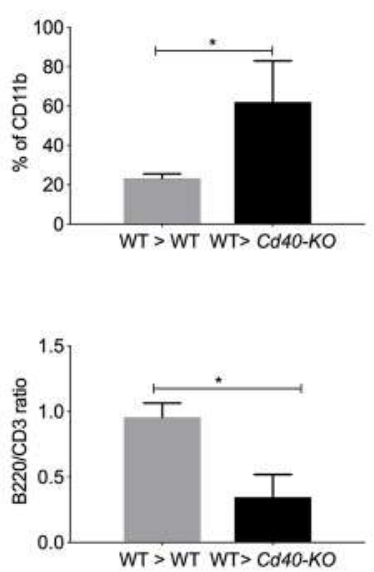

B
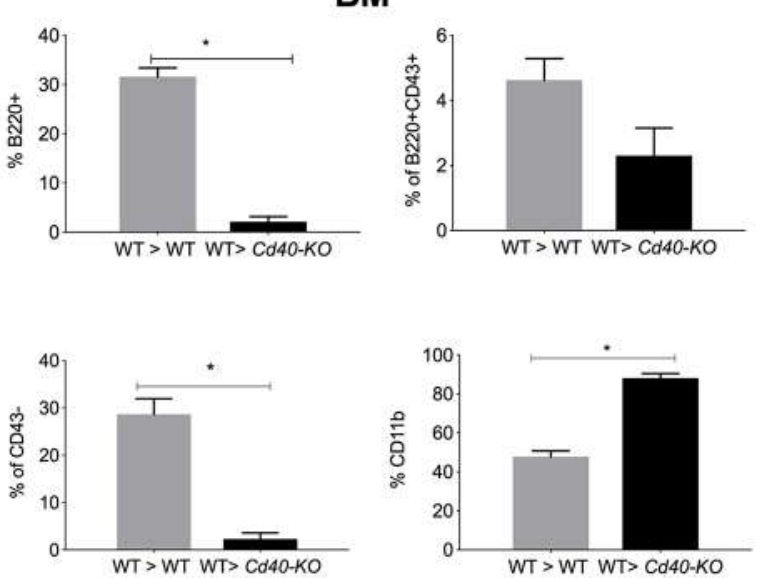

D

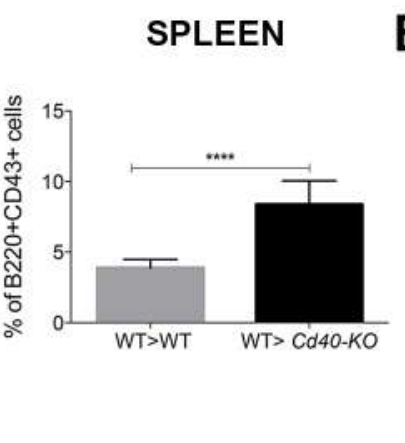

SPLEEN (CD93+ fraction)

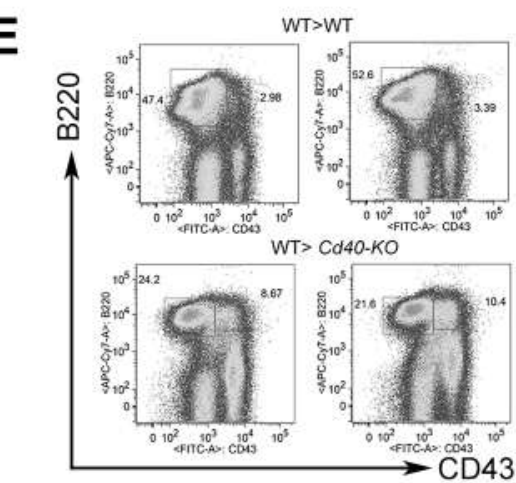

H SPLEEN: MZB, FoB
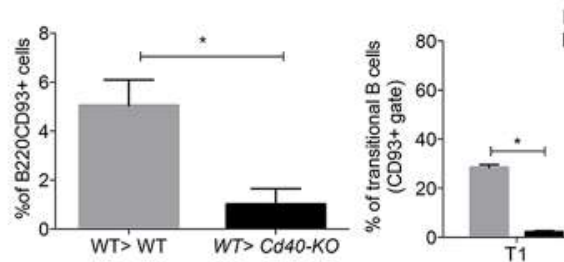

Din $w>W T$

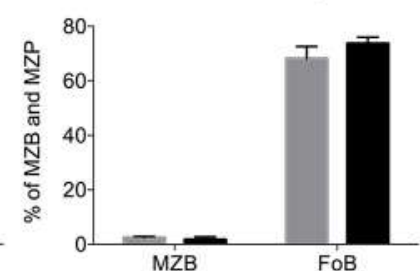

J
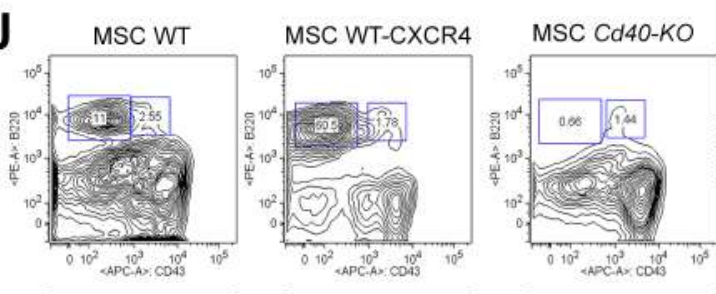

MSC Cd4O-KO-CXCR4
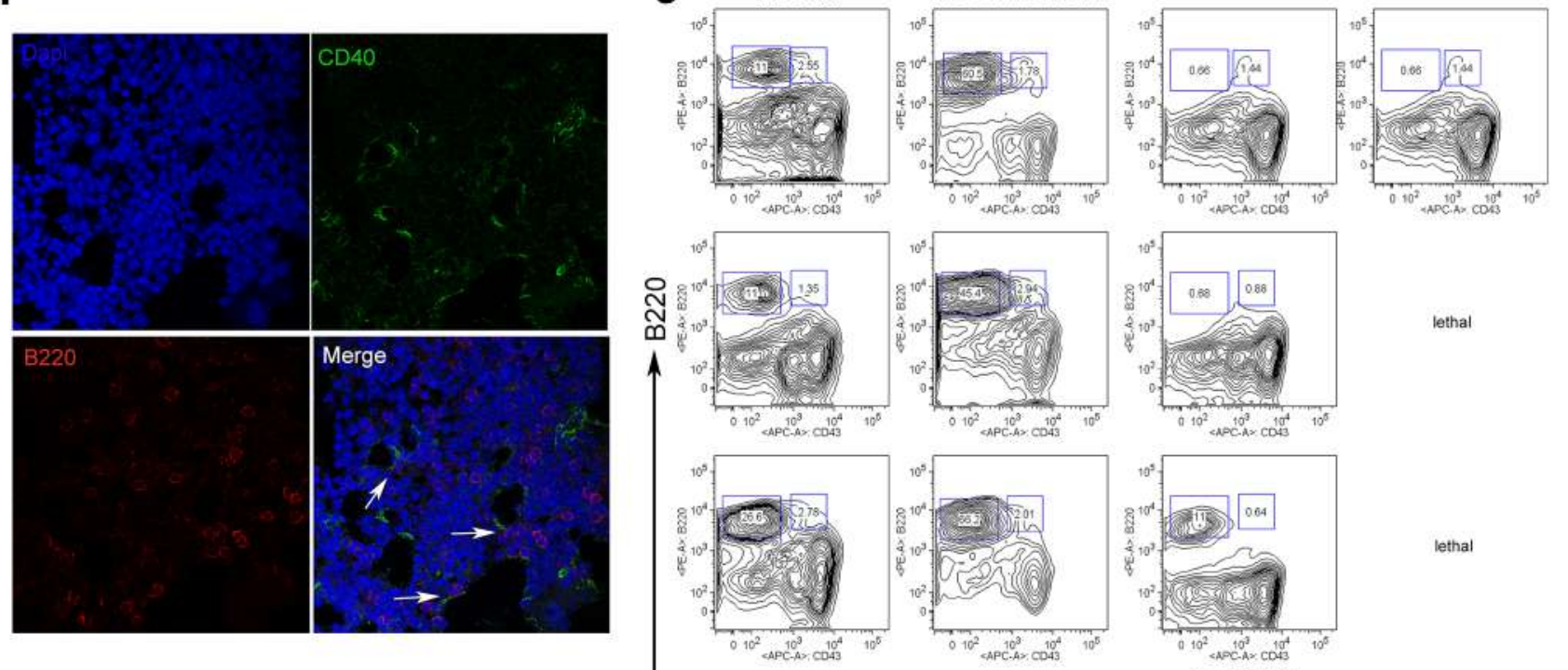

lethal

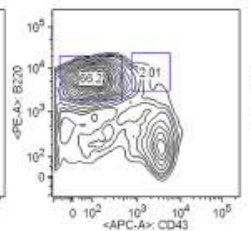

letha!

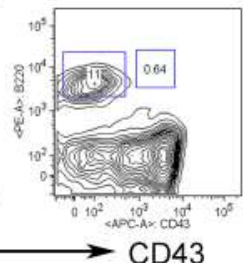


Figure 3

A

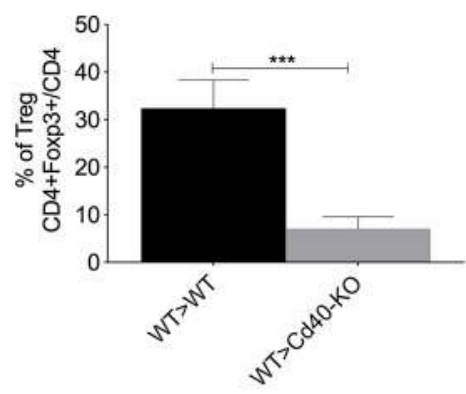

D

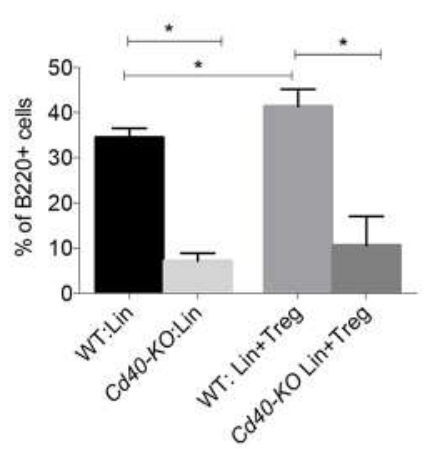

G

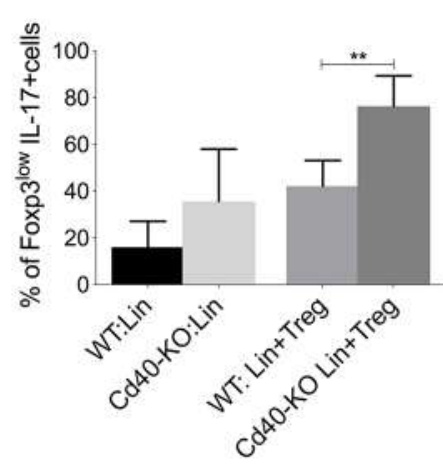

I

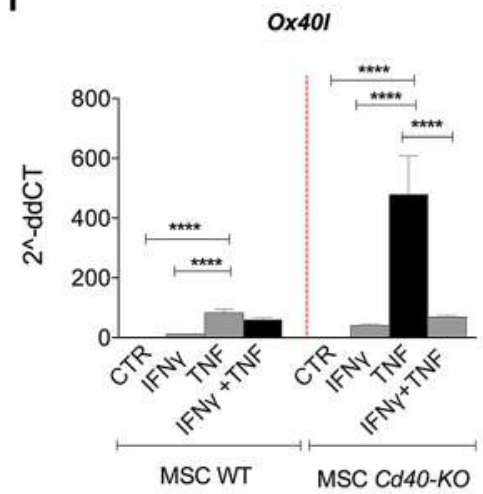

B

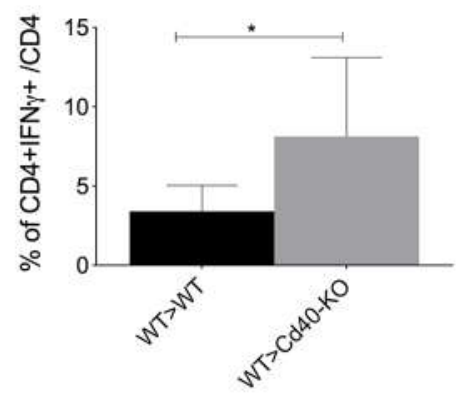

E

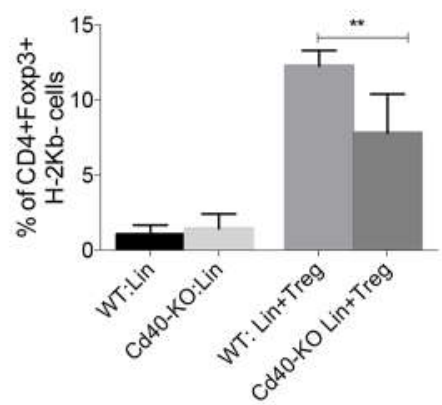

$\mathrm{H}$
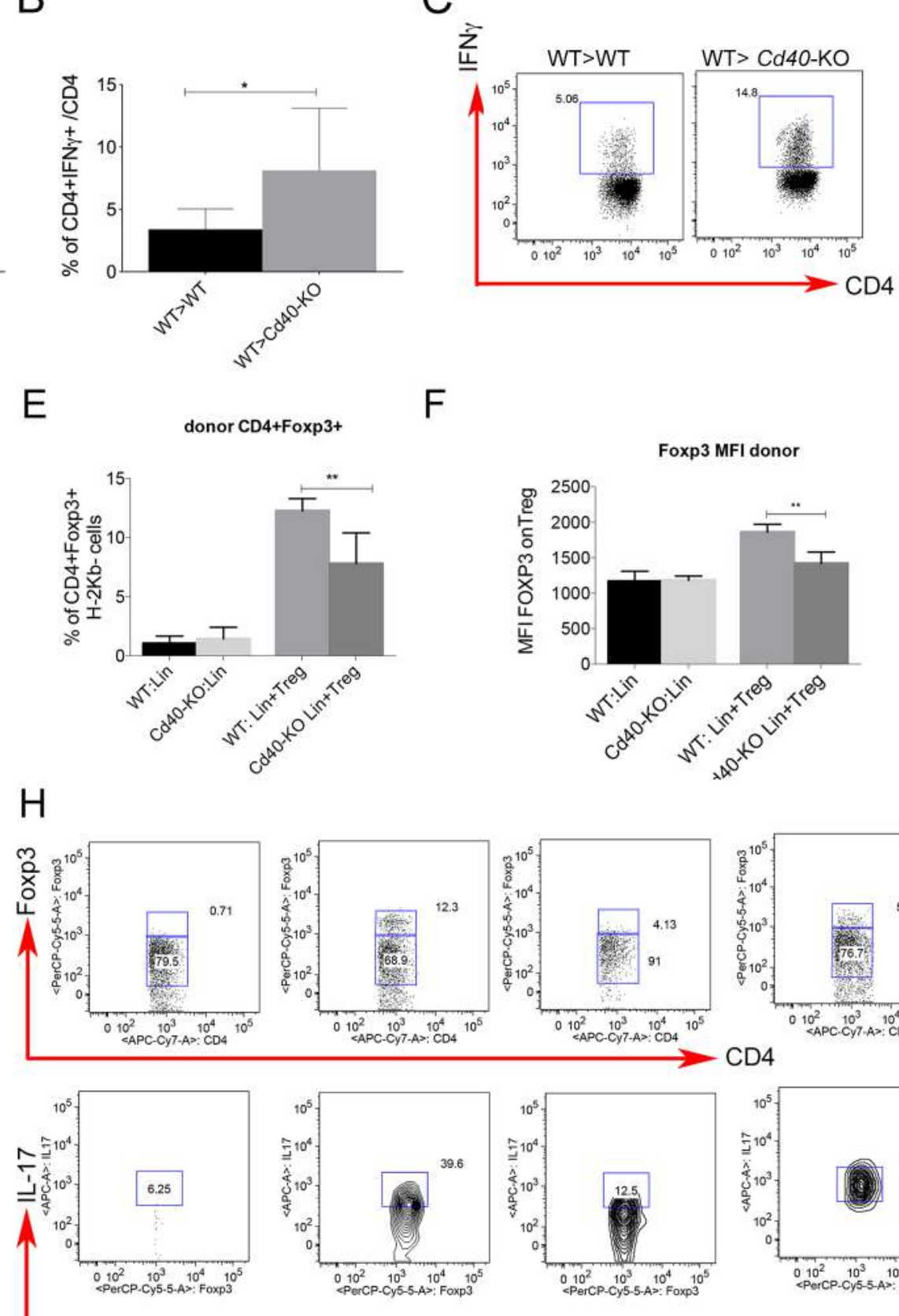

F
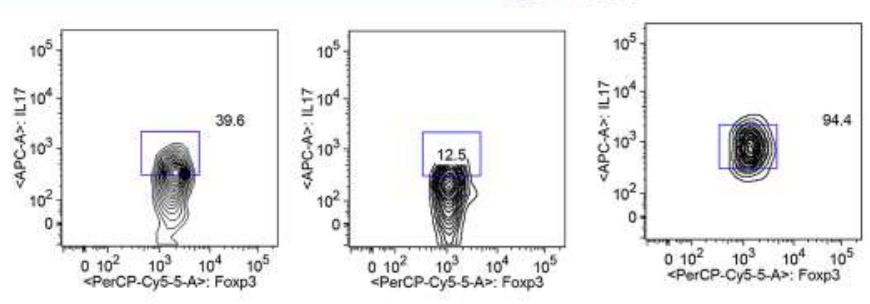

Foxp3

J

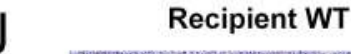

Recipient Cd40-KO
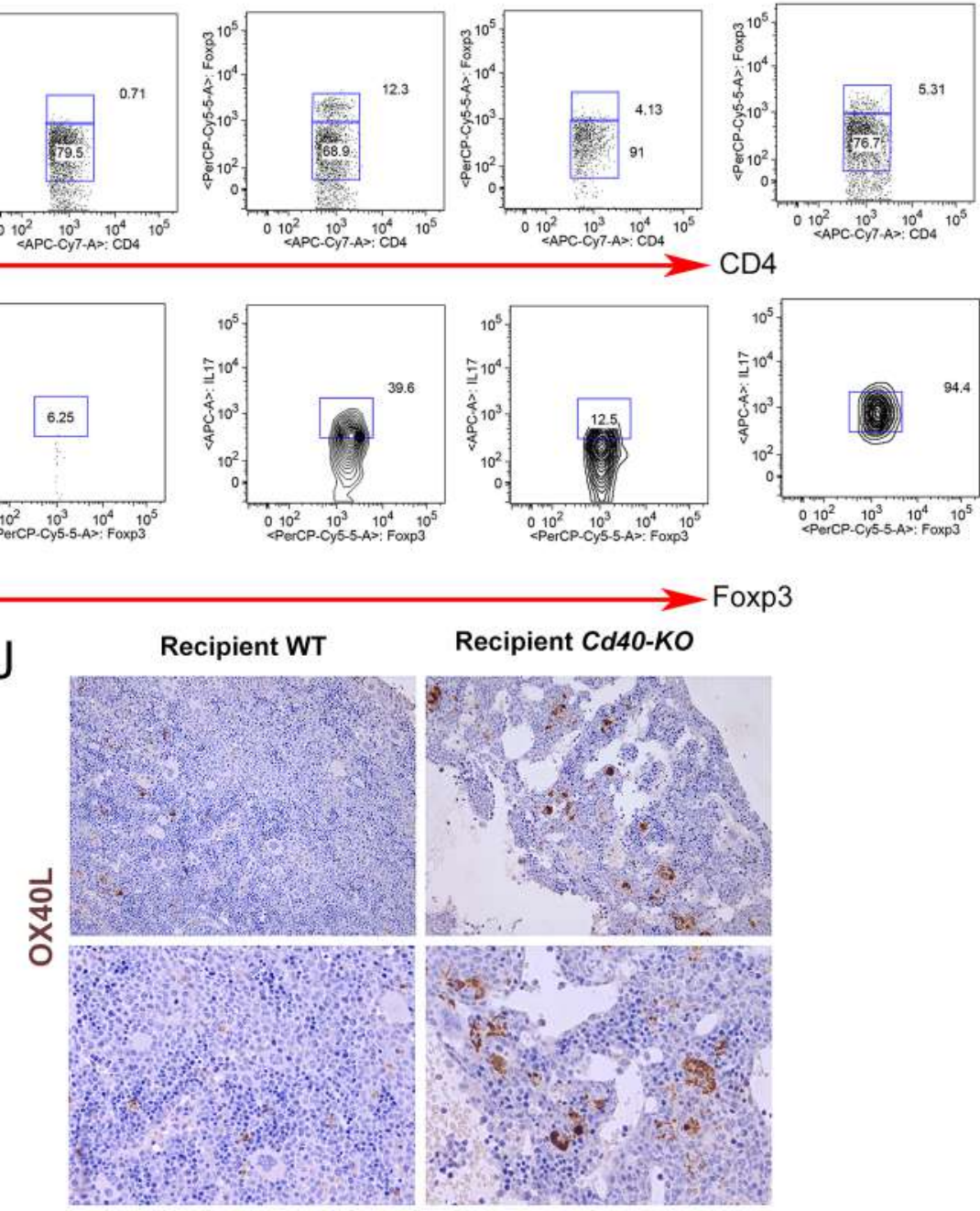
bioRxiv preprint doi: https://doi.org/10.1101/2020.08.10.243691; this version posted August 10, 2020. The copyright holder for this preprint

(which was not certified by peer review) is the author/funder, who has granted bioRxiv a license to display the preprint in perpetuity. It is made available under aCC-BY-NC-ND 4.0 International license.

Figure 4

A

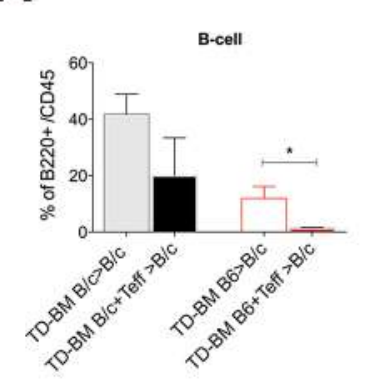

CD8+ Teff

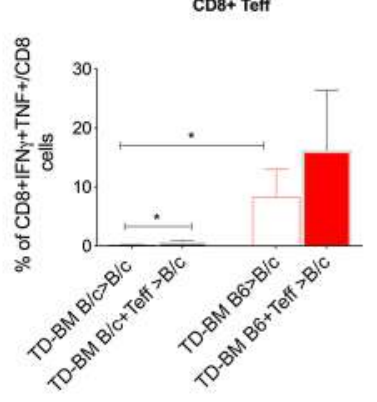

D

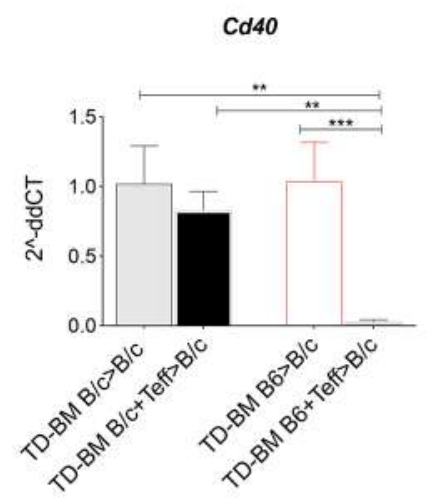

G
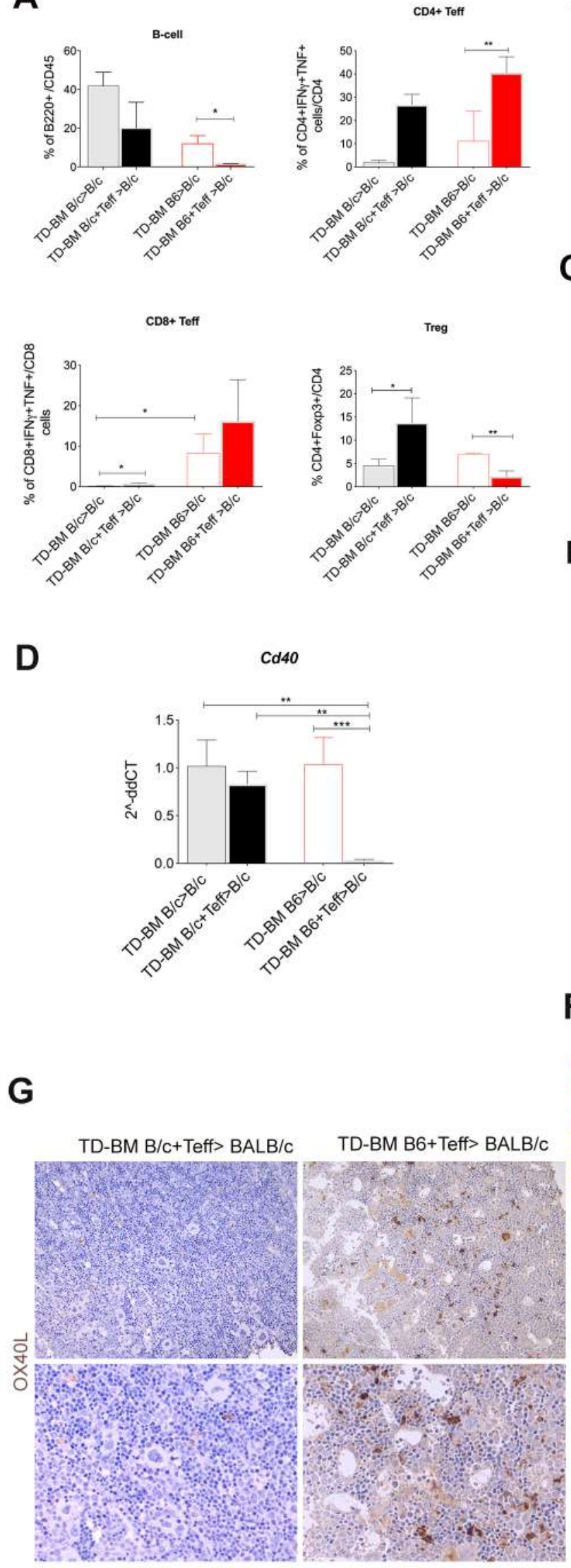

B

C

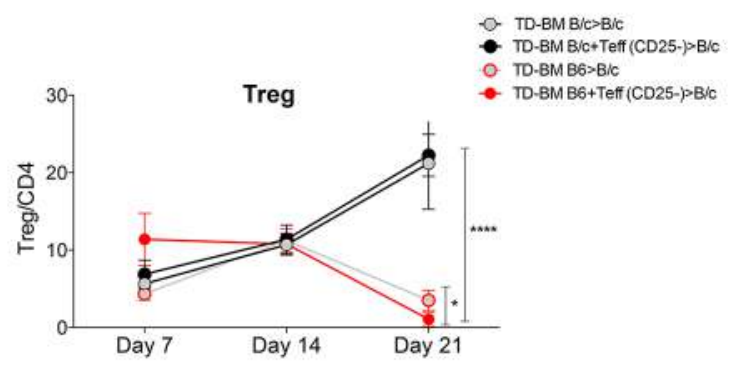

E

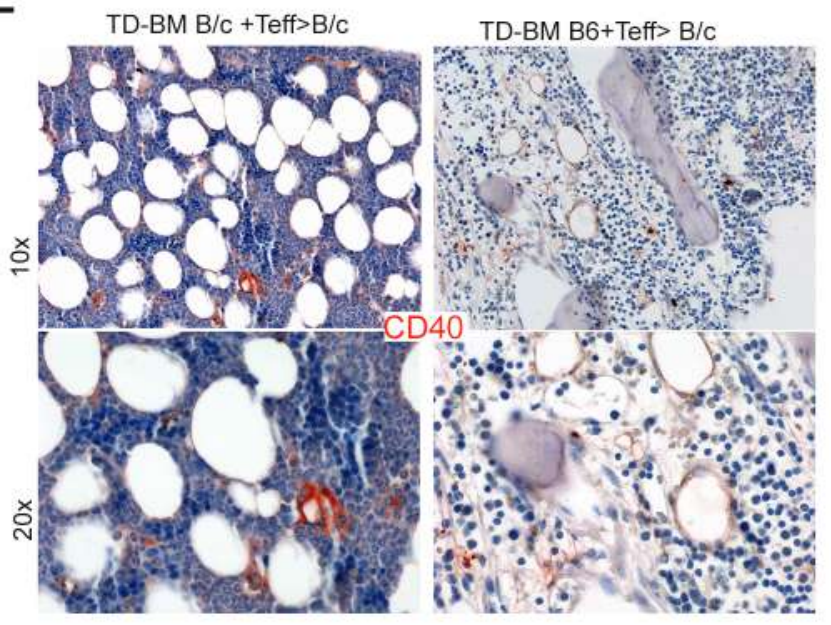

F

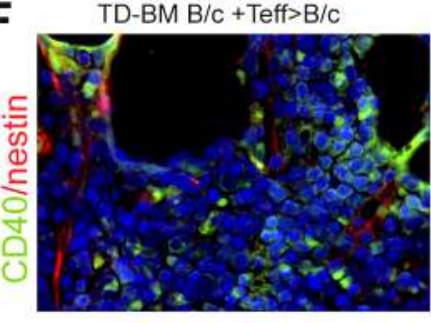

TD-BM B6 + Teff>B/C

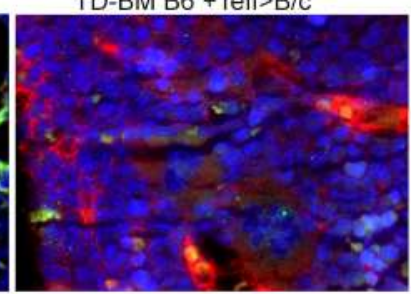

H
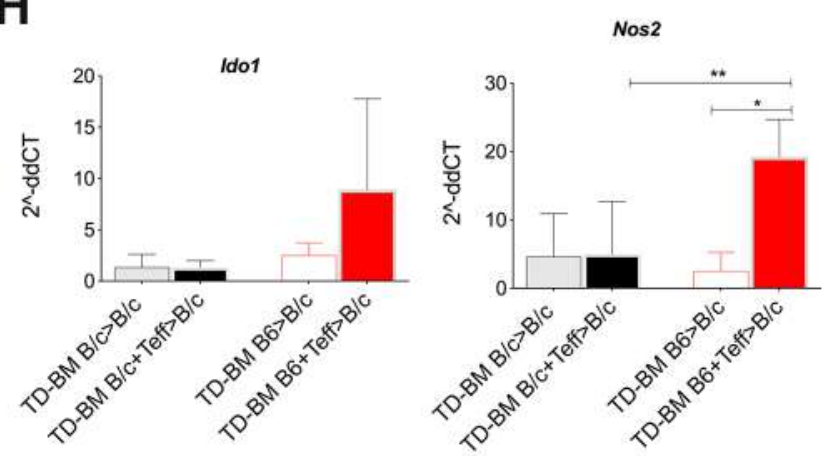
bioRxiv preprint doi: https://doi.org/10.1101/2020.08.10.243691; this version posted August 10, 2020. The copyright holder for this preprint

(which was not certified by peer review) is the author/funder, who has granted bioRxiv a license to display the preprint in perpetuity. It is made available under aCC-BY-NC-ND 4.0 International license.

A
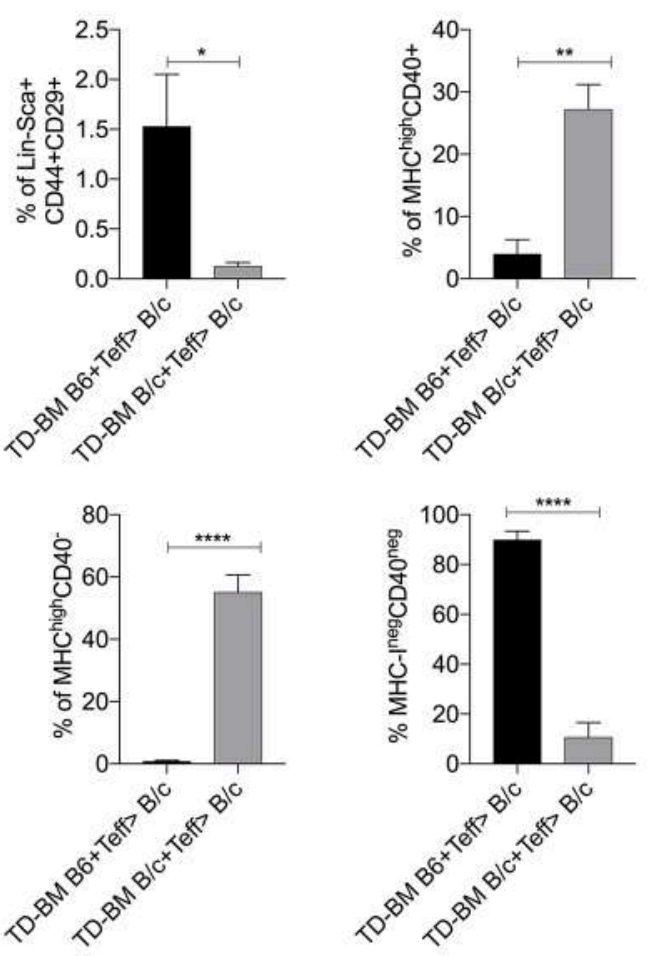

C

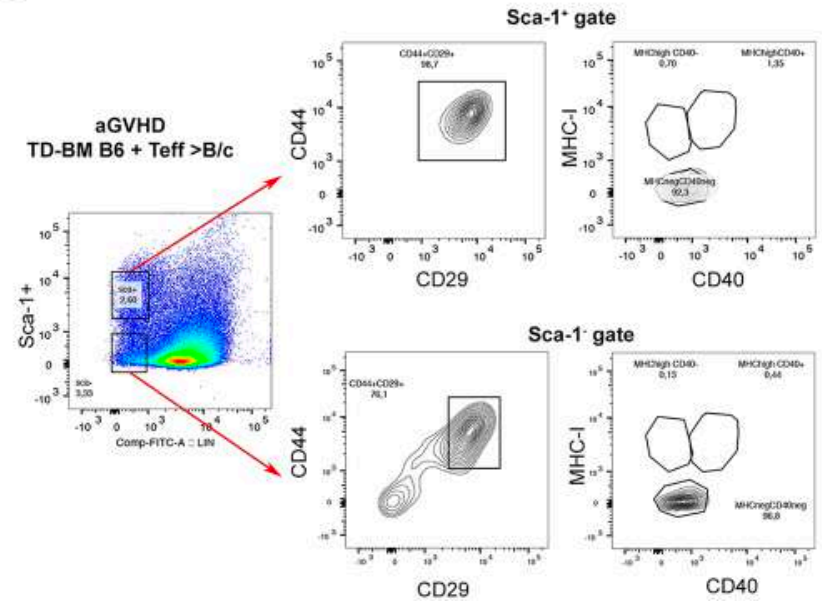

B

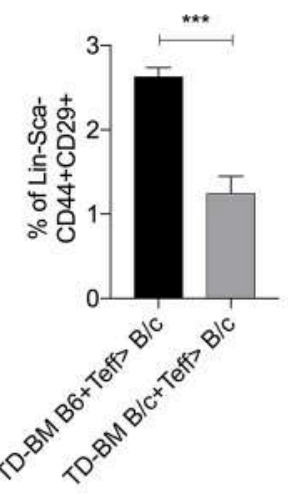

Sca-1-gate
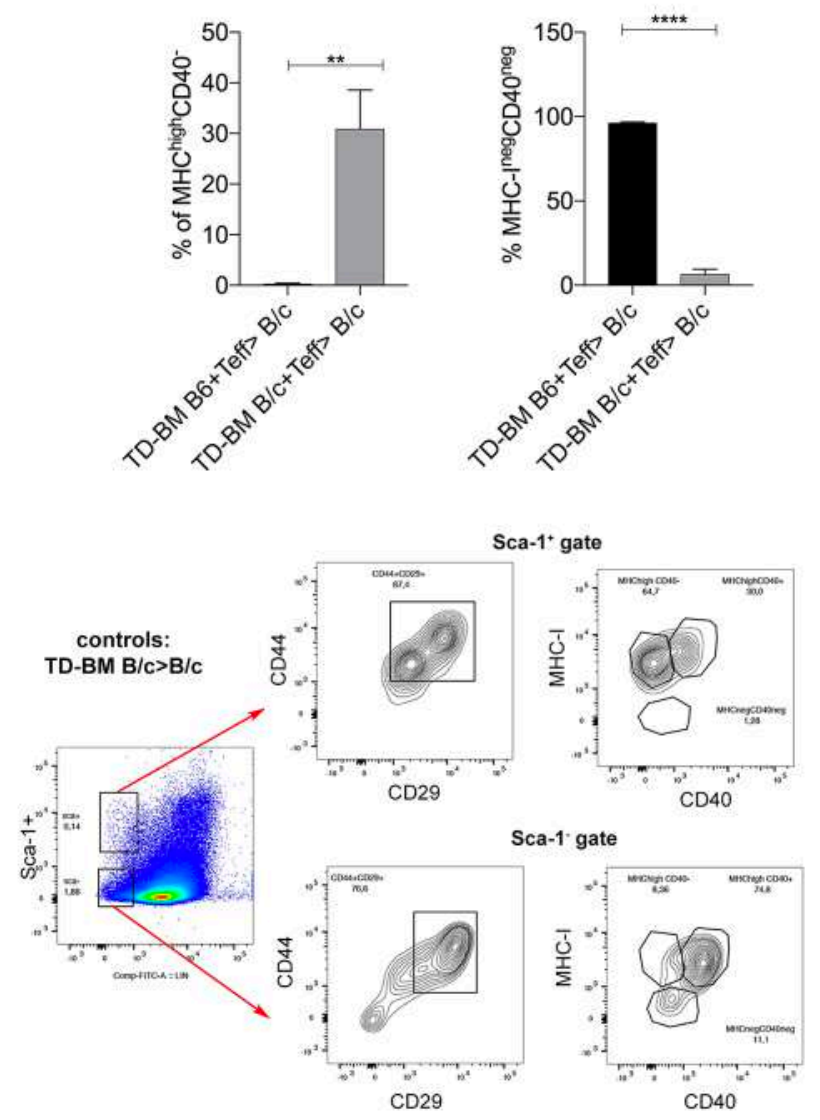
bioRxiv preprint doi: https://doi.org/10.1101/2020.08.10.243691; this version posted August 10, 2020. The copyright holder for this preprint (which was not certified by peer review) is the author/funder, who has granted bioRxiv a license to display the preprint in perpetuity. It is made available under aCC-BY-NC-ND 4.0 International license.

Figure 6

A
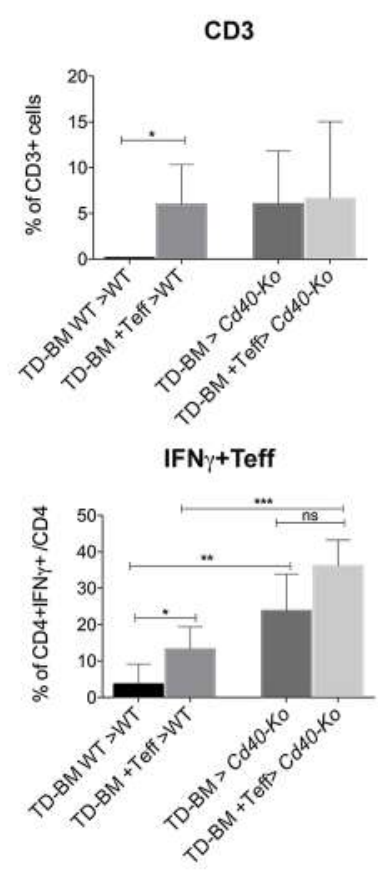

B

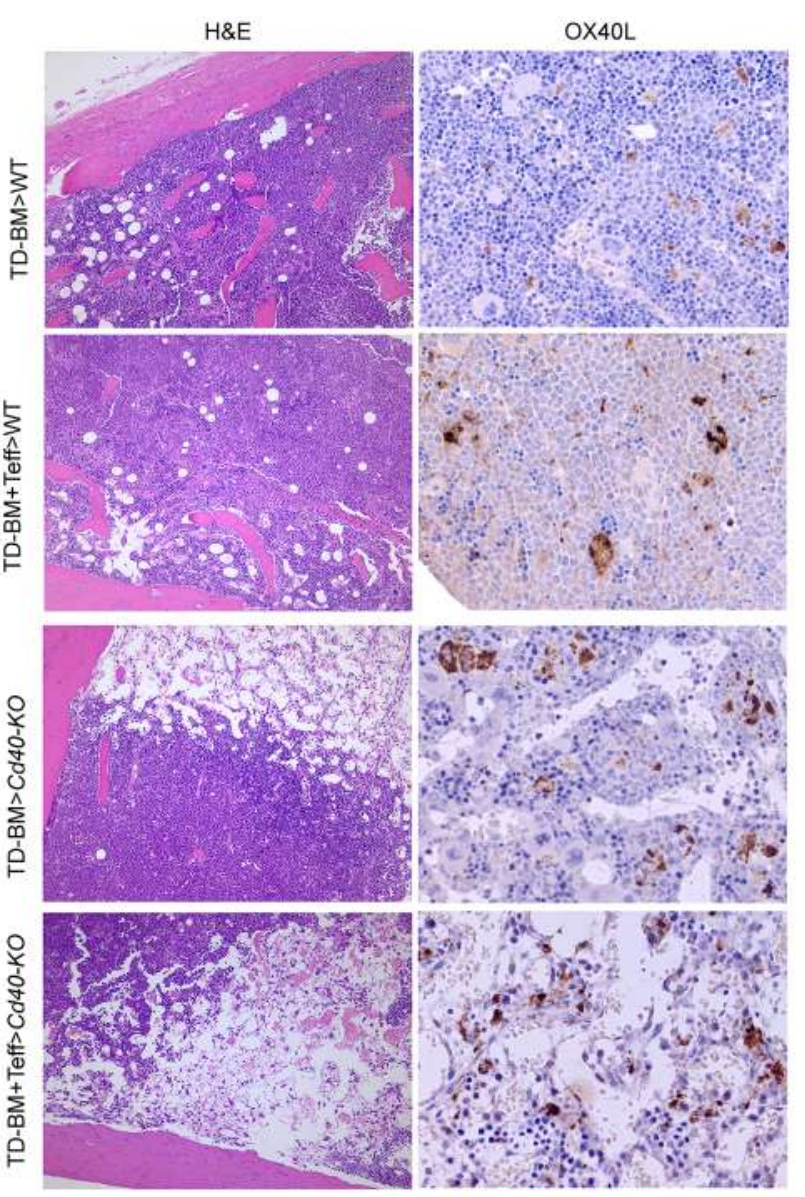


bioRxiv preprint doi: https://doi.org/10.1101/2020.08.10.243691; this version posted August 10, 2020. The copyright holder for this preprint

(which was not certified by peer review) is the author/funder, who has granted bioRxiv a license to display the preprint in perpetuity. It is made available under aCC-BY-NC-ND 4.0 International license.

A

Mesenchymal + hematopoietic CD40

Hematopoietic CD40

Figure 7
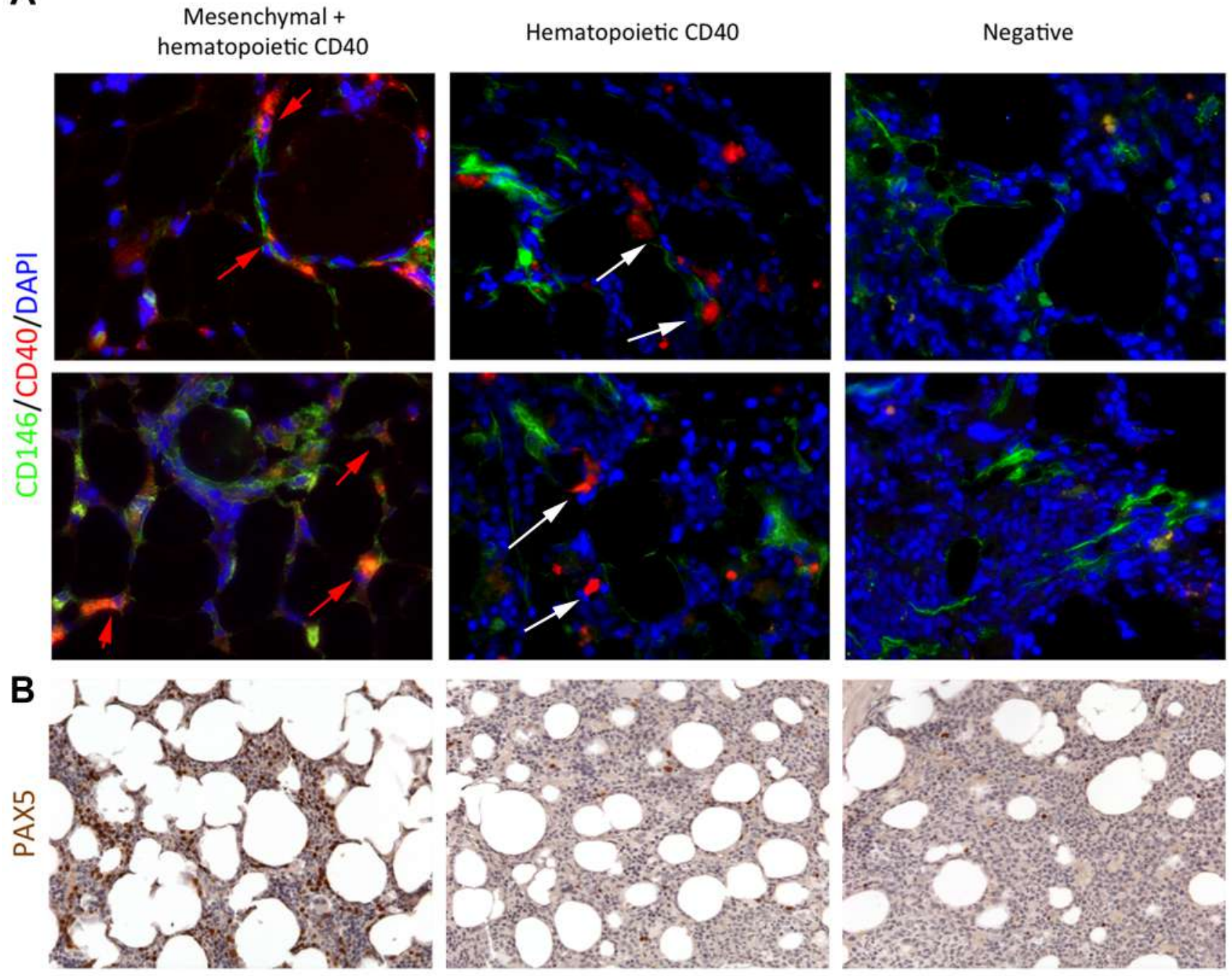

C

nestin/granzyme B/dapi
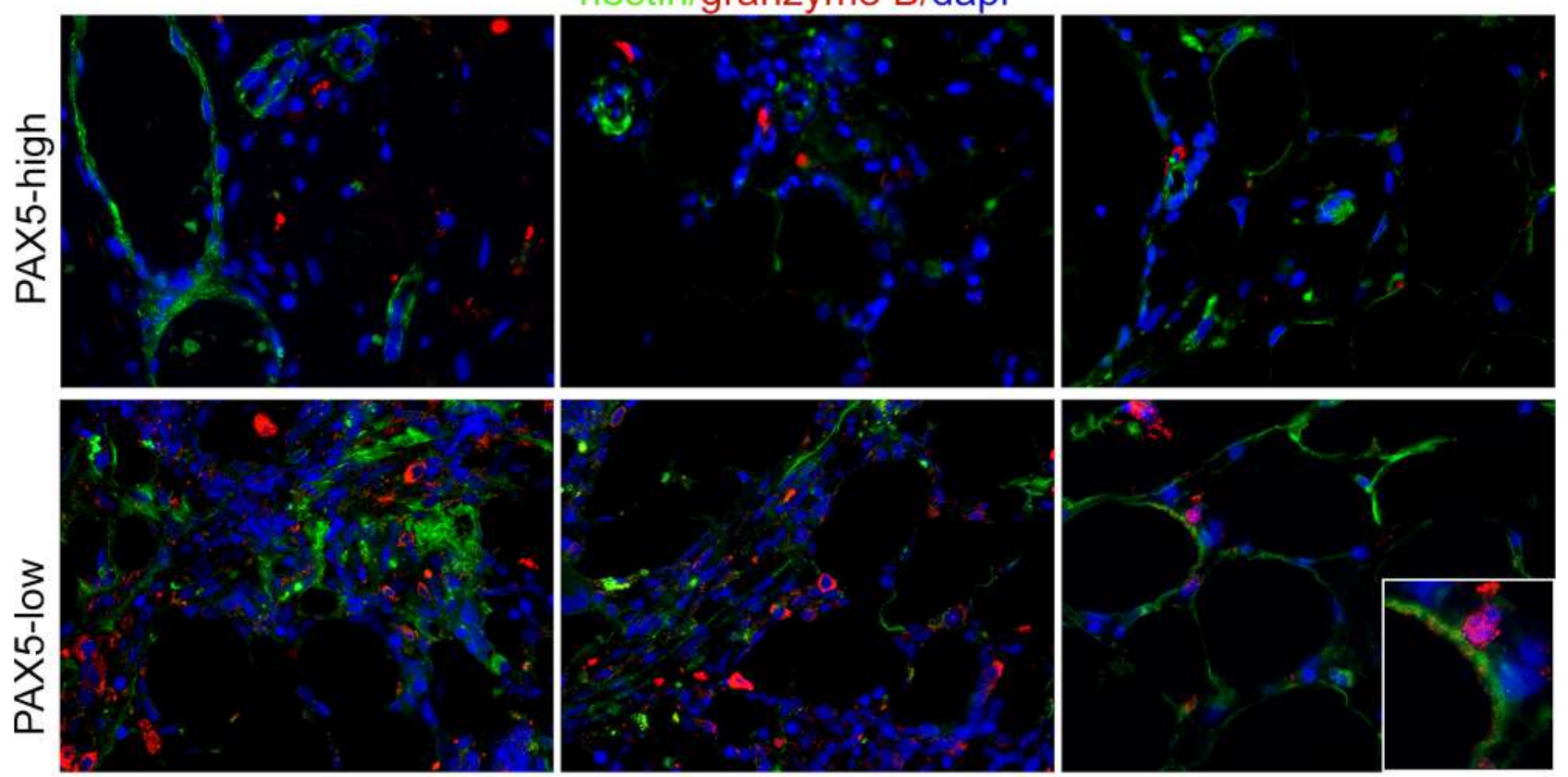